\title{
Murine Staphylococcus aureus chronic infection is cured by theory-driven therapy
}

\author{
Lito A. Papaxenopoulou ${ }^{1}$, Gang Zhao ${ }^{1}$, Sahamoddin Khailaie ${ }^{1}$, Konstantinos Katsoulis-Dimitriou ${ }^{2}$, Ingo \\ Schmitz $^{2,3}$, Eva Medina ${ }^{4}$, Haralampos Hatzikirou ${ }^{1, *}$ and Michael Meyer-Hermann ${ }^{1,5,6, *}$ \\ ${ }^{1}$ Department of Systems Immunology and Braunschweig Integrated Centre of Systems Biology, Helmholtz \\ Centre for Infection Research, Rebenring 56, 38106 Braunschweig, Germany \\ ${ }^{2}$ Institute for Molecular and Clinical Immunology, Medical Faculty, Otto-von-Guericke-University, Leipziger \\ Straße 44, 39120, Magdeburg, Germany \\ ${ }^{3}$ Systems-oriented Immunology and Inflammation Research Group, Department of Experimental Immunology, \\ Helmholtz Centre for Infection Research, Inhoffenstraße 7, 38124 Braunschweig, Germany \\ ${ }^{4}$ Department of Infection Immunology, Helmholtz Centre for Infection Research, Inhoffenstraße 7, 38124, \\ Braunschweig, Germany \\ ${ }^{5}$ Institute for Biochemistry, Biotechnology and Bioinformatics, Technische Universität Braunschweig, \\ Spielmannstraße 7, 38106 Braunschweig, Germany \\ ${ }^{6}$ Centre for Individualised Infection Medicine (CiiM), Feodor-Lynen-Straße 15, 30625, Hannover, Germany.
}

* Corresponding authors: Haralampos Hatzikirou: haralampos.hatzikirou@ theoretical-biology.de; Michael Meyer-Hermann: mmh@theoretical-biology.de.

The authors declare that there is no conflict of interests.

\begin{abstract}
Staphylococcus aureus (S. aureus) is a challenging human pathogen due to its ability to evade the immune system and resist multidrug antibiotics. These evasive strategies lead to chronic and recurrent infections. Many studies have documented that during chronic infections Myeloid Derived Suppressor Cells (MDSCs) exert immunosuppressive mechanisms on T cells. A mathematical model explains how the steady state of chronic infection can be disturbed and suggests therapeutic strategies to clear the infection. Model-driven suggestions were tested experimentally and confirmed complete clearance of $S$. aureus chronic infection.
\end{abstract}

\section{Keywords}

Myeloid Derived Suppressor Cells, MDSCs, Staphylococcus aureus, chronic infection, mathematical model, therapy, cure, heat-killed cells

Staphylococcus aureus (S. aureus) is a bacterial human pathogen colonizing $20 \%$-30\% of the world population and responsible for the genesis of nosocomial-acquired and community-acquired bacterial infections. Colonization by $S$. aureus is typically asymptomatical, implying an equilibrated state between host and bacterium. However, the bacterium can become opportunistic often post-surgery or after implantation of medical devices and can cause skin and soft tissue infections, such as dermatitis, impetigo, and cellulitis ${ }^{1}$, as well as life-threatening conditions like pneumonia and chronic osteomyelitis ${ }^{2}$. Additionally, individuals with immune deficiencies are more susceptible to $S$. aureus infections. The pathogen constitutes a serious problem in clinics worldwide because it uses multiple mechanisms to persist in the host. These include strategies of bacterial 
evasion, multi-drug antibiotic resistance, immunosuppression ${ }^{3}$ or manipulation of the host's immune regulatory mechanisms ${ }^{4 ; 5}$ which lead to chronic and difficult-to-treat infections.

Typically, immunosuppression is achieved via regulatory $\mathrm{T}$ cells (Tregs), $\mathrm{T}$ cell lysis, regulatory B cells (Bregs) ${ }^{6}$, and Myeloid-Derived Suppressor Cells (MDSCs). In the case of S. aureus chronic infections, immunosuppression is not attributed to Bregs, tolerogenic dendritic cells, nor Tregs ${ }^{7}$. Treg-depletion has only a minor effect, whereas T-cell proliferation remains inhibited despite the absence of $\mathrm{B} 220^{+}$and CD11c ${ }^{+}$cells ${ }^{7}$. Nevertheless, T-cell suppression in chronically infected mice has been associated with the expansion of monocytic-like $\left(\mathrm{CD} 11 \mathrm{~b}^{+} \mathrm{Ly}_{6 \mathrm{C}^{+}} \mathrm{Ly}_{6 \mathrm{G}}{ }^{\text {low }}\right.$ phenotype), neutrophilic-like $\left(\mathrm{CD} 11 \mathrm{~b}^{+} \mathrm{Ly}_{6 \mathrm{C}^{\text {low }}} \mathrm{Ly}_{6 \mathrm{G}^{+}}\right.$phenotype) and eosonophiliclike $\left(\mathrm{CD} 11 \mathrm{~b}^{+} \mathrm{Ly} 6 \mathrm{C}^{\text {low }} \mathrm{Ly} 6 \mathrm{G}^{\text {low }}\right.$ phenotype) MDSCs $7 ; 8 ; 9 ; 10 ; 11$, affirming the dominant immunosuppressive role of MDSCs during chronic $S$. aureus infections.

MDSCs constitute a heterogeneous population of immature myeloid cells, which exert their suppressive effect on $\mathrm{T}$ cells by producing reactive oxygen species, nitric oxide, arginase, and inducible nitric oxide synthase. Significant MDSC expansion and the consequent immunosuppressive effect were reported in long-lasting pathological conditions, such as chronic bacterial and viral infections ${ }^{12 ; 13 ; 14}$, cancer ${ }^{15 ; 16}$, and autoimmunity ${ }^{17}$.

In this study we focused on the impact of immunosuppression on the outcome of a $S$. aureus chronic infection. Our aim was to investigate, how a $S$. aureus chronic infection can be fully resolved. Given the systemic MDSC-mediated suppression on T cells ${ }^{18}$ and the fact that MDSC-depletion during a bacterial chronic infection is coupled with depletion of macrophages, and granulocytes, such as neutrophils ${ }^{18}$, it would be challenging to discover therapeutic treatments only by experimental means. However, modelling the chronic infection mathematically could bestow a broader observation of possible treatments that in silico could be expeditiously and cost-effectively tested for rendering sterilizing immunity.

Mathematical models have been used to shed light on issues such as dynamics of $S$. aureus infection and the kinetics of bacterial growth ${ }^{19 ; 20 ; 21}$. Additionally, both deterministic and stochastic mathematical models have been established to illustrate $S$. aureus transmission and antibiotic resistance $22 ; 23 ; 24 ; 25 ; 26 ; 27$. However, there is no mathematical model that illustrates, how a $S$. aureus chronic infection is established and resolved.

Herein, we developed a mathematical model that investigates the impact of MDSC suppressive effects during a $S$. aureus chronic infection aiming to clarify the mechanisms that favour chronicity. Our in silico analysis suggested that triggering an acute inflammation at the state of chronic $S$. aureus infection could perturb the chronic system and eradicate $S$. aureus. Our in silico-driven therapeutic strategy was validated in murine models in vivo by showing complete bacterial eradication.

\section{Results}

\section{Mathematical model suggests therapeutic ideas}

The mathematical model comprises of currently known interactions between bacteria $\mathrm{B}(\mathrm{t})$, $\mathrm{T}$ cells $\mathrm{T}(\mathrm{t})$ and immune regulation mediated by MDSCs. During onset of a chronic infection the existence of bacteria activates immune cells, which proliferate and hinder further growth of bacteria. At the same time, bacteria use various mechanisms to evade immune defenses and continue growing ${ }^{28 ; 29}$. This phenomenon causes incessant activation of the immune responses namely inflammation, a signal that keeps the immune system continuously alert. For prevention of severe injury and tissue damage caused by the constant inflammatory signal, MDSCs get activated and expand systemically ${ }^{18}$ to suppress the T cell activity. The MDSCs have direct contact with the $\mathrm{T}$ cells ${ }^{30}$, hampering the latter from expressing their full aggressive effect on bacteria. Bacteria are therefore not eliminated, but nevertheless cannot grow any further because T cells can still exert on them an extent of suppression. This leads to a non-growth and non-eradication of bacteria, and consequently the establishment of a stable equilibrium between the aforementioned three groups of cells. This equilibrium is generally known as a $S$. aureus chronic infection. A schematic representation of the model is illustrated in Fig. 1A.

\section{Dynamics of chronic state establishment}

To first validate model accuracy and consistency we reproduced the inverse proportional behaviour between $\mathrm{T}$ cells and MDSCs (Fig. S1). Analytical stability analysis and numerical analysis (Supplementary) showed how a $S$. aureus chronic infection is established in the absence of any treatment intervention (Fig. 1B and Fig. S2A). The onset of the infection induces strong inflammation, which activates $\mathrm{T}$ cells. The competition for dominance 
between bacteria and $\mathrm{T}$ cells creates oscillations in the population dynamics (Fig. 1B). When inflammation becomes long-lasting, MDSCs expand and suppress $\mathrm{T}$ cells. This dampens oscillations in both $\mathrm{T}$ cell and bacterial populations. However, increasing accumulation of MDSCs in the lymphoid organs, leads to increasing suppression on T cells $(\Sigma)$, which upon a critical threshold renders the infection non self-curable (Fig. 1D). As a consequence, the system reaches a steady state (Fig. 1C), the chronic infection, where bacteria persist in the host organism but are simultaneously unable to further grow due to their containment by $\mathrm{T}$ cells ${ }^{31}$. Once at this stage, sterilizing immunity can be attained only by using treatments, which destabilize (i.e. perturbate) the stable steady state of chronic infection.

\section{Model-driven therapeutic strategies for Staphylococcus aureus chronic infection}

To explore perturbation strategies that would destabilize the stable state of chronicity, we tested different values of $k_{b}$ and $\Sigma$. These parameters were specifically chosen because they represent T-cell activation and recruitment, and $\mathrm{T}$ cell suppression by MDSCs, respectively, rates that are conventionally seen to play a key role to the establishment of chronic infection. Based on the eigenvalues of the ODE system (Eqs. (1)-(2)), we characterized the steady states as unstable and stable, and divided the separatrix (phase diagram) into cure and chronic infection regimes, respectively (Fig. 2A-2B).

Our next step was to determine the infected mouse's position in the phase diagram to suggest therapeutic strategies. Since not all infected mice are synchronized in the same infection phase, we found all possible positions of the infected organism in the phase diagram. All positions lied into the region of chronic infection (Fig. 2A-2B).

According to the separatrix of cure and chronic infection (Fig. 2A-2B), we concluded that the resolution of chronic infection is achieved by either (a) shifting the infected organism from the chronic infection regime (black area) to the cure regime (green area) or (b) by extending the cure regime itself (Fig. 2B). Relocation of the infected individual from the chronic infection region to cure is achieved by increasing T-cell activation and recruitment $\left(k_{b}\right)$ and/or by decreasing MDSC suppression on T cells ( $\Sigma$ ) (Fig. 2A). Furthermore, expansion of the cure zone (Fig. 2B) is achieved by counter-intuitively increasing the proliferation rate of bacteria $\left(r_{b}\right)$ and/or by reducing bacteria's killing rate via immune cells $\left(c_{b}\right)$. Altogether the model indicates that all four aforementioned perturbation strategies would destabilize the chronic steady state in such way, that resolution of chronic infection, eradication of the bacteria, and sterilizing immunity would be achieved (Figs. 2C, S2B-S2D).

Experimental testing was essential to validate the model predictions. Boosting $k_{b}$ in vivo, as suggested by the in silico predictions (Fig. 2C), could be achieved by introduction of heat-killed (HK) bacteria into the infected organism. The physiological $k_{b}$ increase was incorporated into the model with the addition of a $k_{b} \cdot B_{d}$ term in the T cells' ODE (Methods), where $B_{d}$ the dose of HK injection and $k_{b}$ the activation of immune system from HK bacteria assumed the same as for live bacteria (Table 1). Since our aim was to investigate a $S$. aureus chronic infection, the experimental perturbation had to be carried out after the 13th day of infection. The perturbation with HK bacteria was scheduled on the 14th day after initial infection with $5 \times 10^{7} \mathrm{~S}$. aureus cells. Numerical simulations suggested that the minimum dose needed for cure would be $4 \times 10^{7} \mathrm{HK}$ bacteria (Fig. S3). For our experiments, we opted for the amount of $10^{8} \mathrm{HK}$ cells.

To identify, on which day the infected mice would recover from infection and perform the sampling, we followed the simulations' results, which predicted sterilizing immunity on day 34.5 post-infection (Fig. 2C). However, taking into account the corresponding stochasticity of a biological system, the experimental sampling was set on the 37th day post-infection (Fig. 2C). 
A
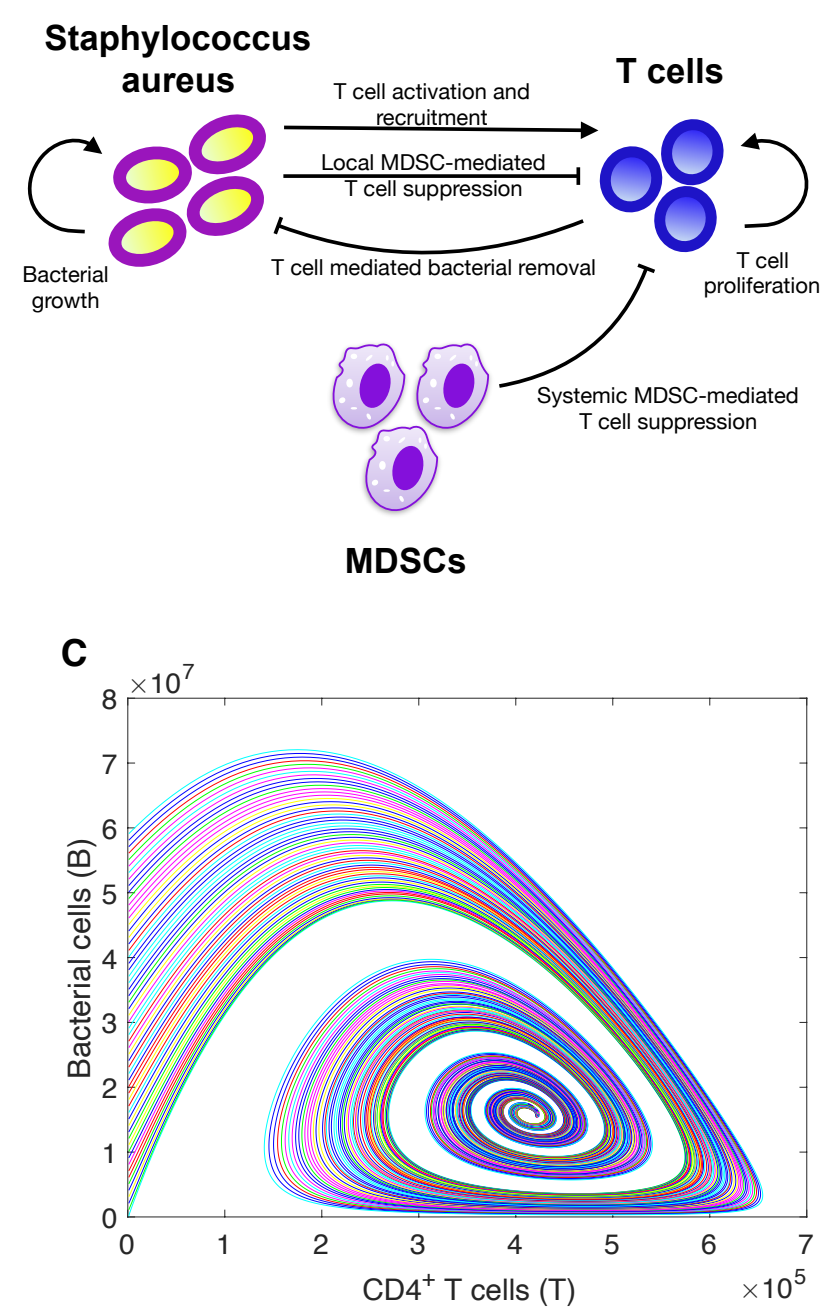

B

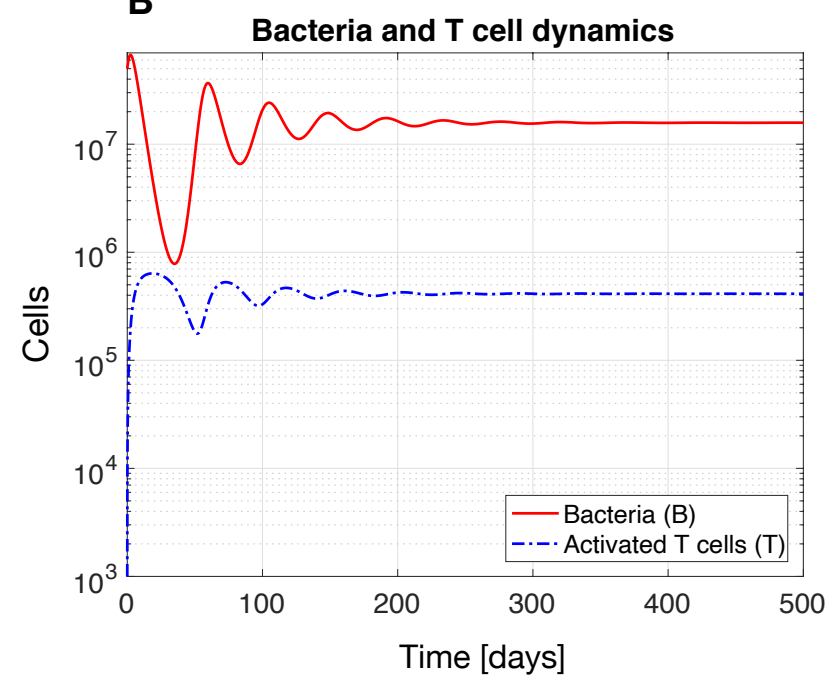

D

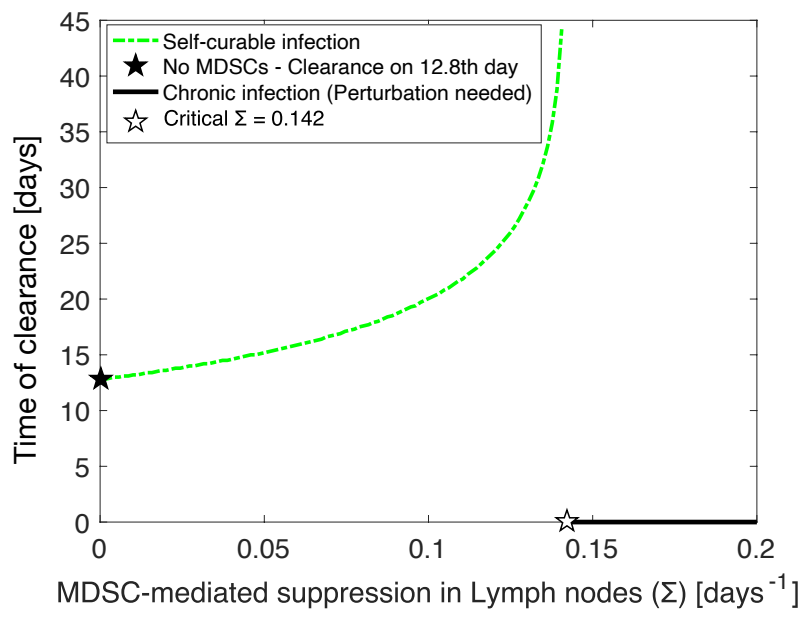

Figure 1: Establishment of $S$. aureus chronic infection. (A) Schematic representation of staphylococcal chronic infection model. After initial infection $S$. aureus cells proliferate in the host as $r_{b} B(t)(1-B(t) / \kappa)$. Bacteria activate T cells as $k_{b} B(t)$, which proliferate as $r_{t} T^{2}(t)$ and suppress bacteria as $c_{b} T(t) B(t)$. Once the bacterial infection becomes chronic, strong MDSC suppression on T cells takes place either locally as $c_{T} B(t) T(t)$ or systemically as $\Sigma \cdot T(t)$. (B) Initiation of the infection in a previously healthy host causes strong inflammation, which rapidly activates $\mathrm{T}$ cells. Infection was induced by setting the bacterial population equal to $5 \times 10^{7}$ cells on day 0 in Eq. (1). The oscillatory dynamics of bacteria (B) and activated $\mathrm{T}$ cells (T) in time are shown as numerical solutions of the ODE system (see Methods). (C) The interplay between bacteria and $\mathrm{CD}^{+}{ }^{+} \mathrm{T}$ cells leads to a stable steady state. For changing initial numbers of bacteria in the range $\left[10^{5}, 6 \times 10^{7}\right)$ on day 0 , the system always terminates in the stable equilibrium, which physiologically corresponds to the chronic infection. (D) Infection was initiated as in (B). The day of simulated clearance is shown for increasing values of $\Sigma$ in the range [0, 0.2], physiologically representing MDSC accumulation in the lymphoid organs and incremental T cell suppression by MDSCs. For changing value of the parameter $\Sigma$ in the range [0, 0.2], the ODE solver calculates the bacterial numbers. For bacterial numbers $<0.000001$, the infection is considered cured (dashed line), else persisting (solid line). For resolved infections the corresponding day of clearance is shown, or set to zero for persisting (i.e. chronic) infections. The black star represents the scenario when MDSCs are absent and hence T-cell suppression does not exist $(\Sigma=0)$. The white star represents the critical value of $\Sigma$, when the infection becomes persistent. The values for the rest of the model parameters are as shown in Table 1.

\section{In vivo cure after model-driven perturbation treatment}

Our mathematical model, described previously, incorporates the effect of MDSCs during a S. aureus chronic infection. Its analysis provided perturbation strategies that showed sterilizing immunity in silico. We proceeded to validate our in silico predictions in vivo. For this purpose, C57BL/6 mice were infected intravenously with $S$. 
A

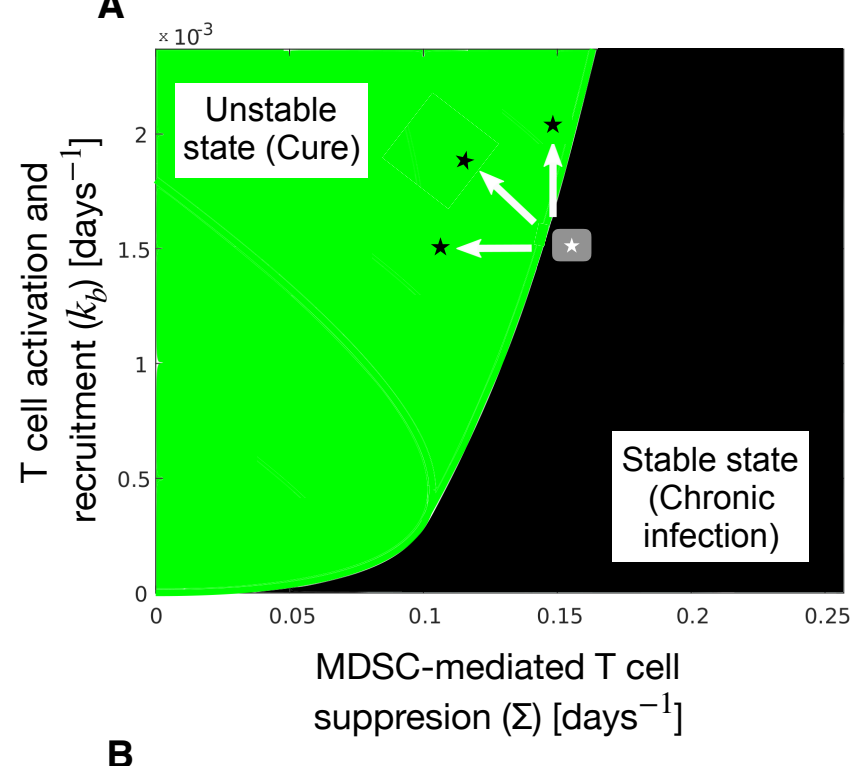

C $k_{b}$ increase on the 14th day of the infection,

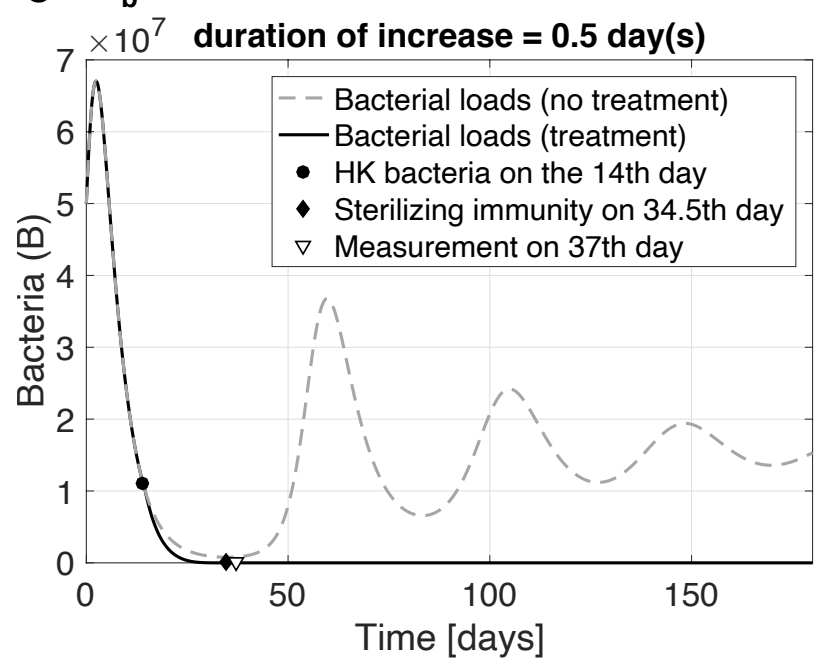

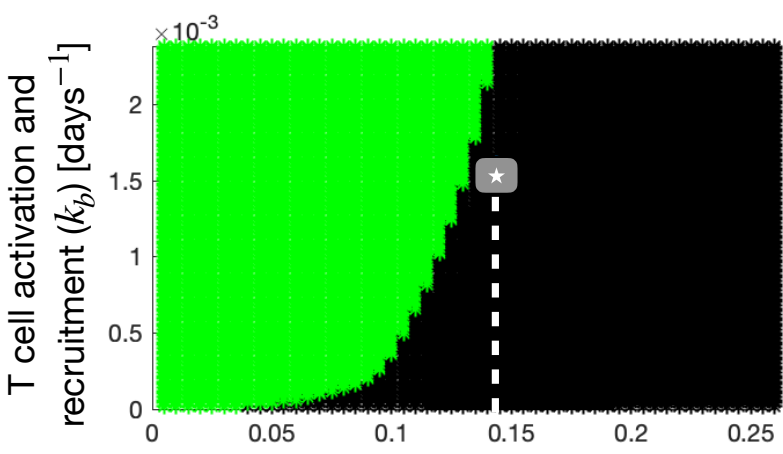

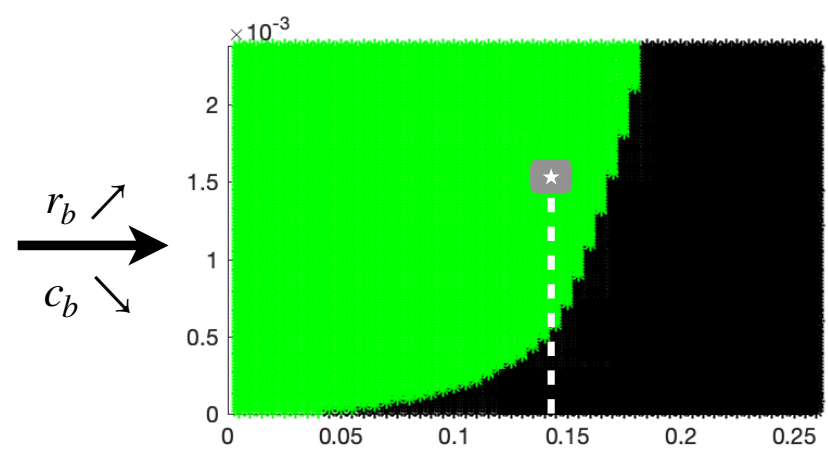

MDSC-mediated T cell suppresion ( $\Sigma$ ) [days ${ }^{-1}$ ]

Figure 2: (A-B) Phase diagram: Escaping from the state of chronic infection. Based on the eigenvalues of the system, the phase diagram was divided into stable state (black) and unstable state (green), which represent the physiological chronic infection and cure, respectively. The white star represents the average position of the infected host during a chronic staphylococcal infection, which was determined by the values of parameters $k_{b}$ and $\Sigma$ as obtained from the fitting results (Table 1). The gray area (cloud) was created using the $95 \%$ confidence intervals of parameters $k_{b}$ and $\Sigma$ as obtained from the fitting results (Table 1) and represents possible positions of the infected mice in the separatrix. (A) Given that the state of infected hosts is as illustrated, it is obvious that mere increase of $\mathrm{T}$ cell activation and recruitment parameter $k_{b}$ and/or decrease MDSC suppression on T cells $\Sigma$ would shift the infected host into the green area of sterilizing immunity (black stars). (B) Increasing the cure regime (green) is achieved by utilizing counter-intuitive therapeutic ideas such as reduction of bacteria's killing rate via immune cells $c_{b}$ and/or increasing the proliferation rate of bacteria $r_{b}$. Expansion of the cure area engulfs the infected host (white star), providing sterilizing immunity. (C) Increase of T-cell activation and recruitment parameter $k_{b}$ confers sterilizing immunity from a $S$. aureus chronic infection in silico. Re-stimulation of the immune system is induced by administering $10^{8} \mathrm{HK}$ cells on the 14th day post-infection for a perturbation window that lasts half day (see Methods). Infection was induced by setting the bacterial population equal to $5 \times 10^{7}$ cells on day 0 in Eq. (1).

aureus strain SH1000. On the 14th day post-infection mice received intraperitoneal injection with HK bacteria of $S$. aureus strain SH10000 (Fig. 3A).

In a previous study ${ }^{31}$, it was shown that when C57BL/6 mice were infected with $S$. aureus, bacteria were progressively depleted from multiple organs and persisted only in the kidneys. Therefore bacterial load quantification was performed in mice's kidneys following the mathematical model's predictions (Fig. 2C). 


\section{Complete bacterial clerance after heat-killed bacteria perturbation treatment}

Sampling on the 37th day post-infection validated the model's predictions. All mice, which had received the perturbation of HK S. aureus cells, achieved sterilizing immunity from S. aureus chronic infection (Fig. 3B). In contrast, the majority of control mice, which had only received PBS instead of HK bacteria were still infected with high bacterial burden (Fig. 3B).

\section{Recovery of $T$ cell function}

Given the fact that progression of a $S$. aureus infection from acute to chronic renders $\mathrm{T}$ cells anergic ${ }^{31}$, our next step was to check the proliferative response of spleen $\mathrm{T}$ cells. Our results indicated that spleen $\mathrm{T}$ cells from treated mice were hyper-responsive to stimulation with anti-CD3 and anti-CD28 antibodies and actively proliferated (Fig. 3D). However, T cells of infected control mice, which had received PBS instead of HK bacteria, exhibited unresponsiveness to TCR re-stimulation (Fig. 3D).

\section{Reduction of MDSCs after heat-killed bacteria perturbation}

Interestingly, we found that the perturbation of the chronic system with HK bacteria did not only boost $\mathrm{T}$ cell function, but also aided in MDSC abatement. Flow cytometry revealed significant reduction of all MDSC subsets in mice's spleens, which had received HK bacteria (Fig. 3F). Reduction of MDSCs after treatment with heatkilled $S$. aureus was coupled with p-value of $0.0220(*)$ and $0.0004(* * *)$ for monocytic-like (M-MDSC) and neutrophilic-like (PMN-MDSC) MDSCs, respectively.

\section{Perturbation with Streptococcus pyogenes results in sterilizing immunity in half of the infected mice's population}

To further elucidate whether the HK perturbation strategy elicits antigen-specific responses or not, we repeated the aforementioned experiments, initially infecting with $S$. aureus and treating with HK Streptococcus pyogenes (S. pyogenes) cells (Fig. 3A). In these experiments, measurements revealed complete clearance in $50 \%$ of the infected mice (Fig. 3C). By contrast, the majority of control mice remained infected. Additionally, T cells responded to stimulation with anti-CD3 and anti-CD28 antibodies only in cured mice (Fig. 3E). Nevertheless, flow cytometry illustrated reduction of MDSCs subsets in all HK-treated mice (Fig. 3G). Reduction of MDSCs after treatment with heat-killed $S$. pyogenes was coupled with p-value of $0.0296\left(^{*}\right)$ and $0.0212(*)$ for monocyticlike (M-MDSC) and neutrophilic-like (PMN-MDSC) MDSCs, respectively.

Some diversity in the bacterial loads of control mice in all experiments was observed (Fig. 3B and 3C). All control mice (19 in total) were infected by $S$. aureus and only received PBS instead of the heat-killed treatment. It was observed that in five of them $S$. aureus was eradicated. Such behaviour is occasionally observed due to individual variation during the innate immune response in the acute phase of the infection.

To comprehend why half of infected mice were cured after HK $S$. pyogenes treatment (in contrast to HK $S$. aureus treatment), we simulated the HK dose and estimated day of clearance for different values of immunostimulatory parameter $k_{b}$. HK $S$. pyogenes cells stimulate the T cells to a lesser extent than the HK treatment with $S$. aureus cells, since the antigen for the latter had been encountered in the host upon initial infection. Our simulations indicated that $100 \%$ clearance of bacteria until the sampling day can possibly be conferred when higher S. pyogenes HK dose is administered (Fig. S4).

\section{HK treatments during established $S$. aureus chronic infection induce strong acute inflammation}

Our experiments in vivo verified that the HK injection initiates acute inflammation during the chronic establishment of $S$. aureus infection. In particular, peritoneal exudates were sampled 12 hours after HK treatment with $S$. aureus or $S$. pyogenes and showed massive increase in amounts of $\mathrm{CD} 11 \mathrm{~b}^{+} \mathrm{Ly} 6 \mathrm{C}^{+}$monocytes and $\mathrm{CD}_{11} \mathrm{~b}^{+} \mathrm{Ly}_{6 \mathrm{G}}{ }^{+}$granulocytes (neutrophils) in $\mathrm{HK}$ treated mice in comparison to the control mice, which had only received PBS (Fig. S5). Interestingly, in case of HK S. aureus treatment, which cured all infected hosts, 


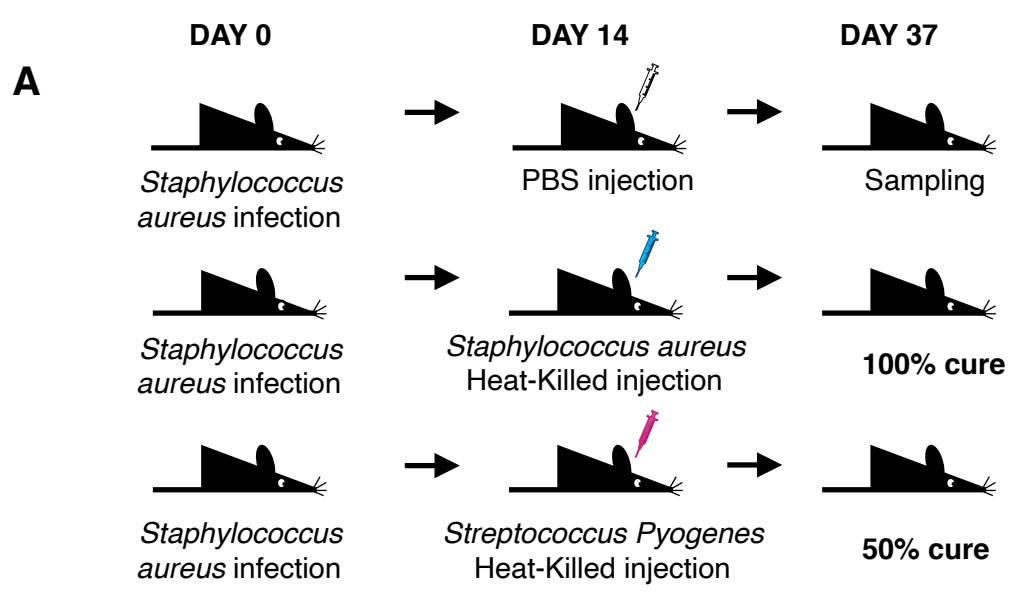

B HK treatment with S. aureus
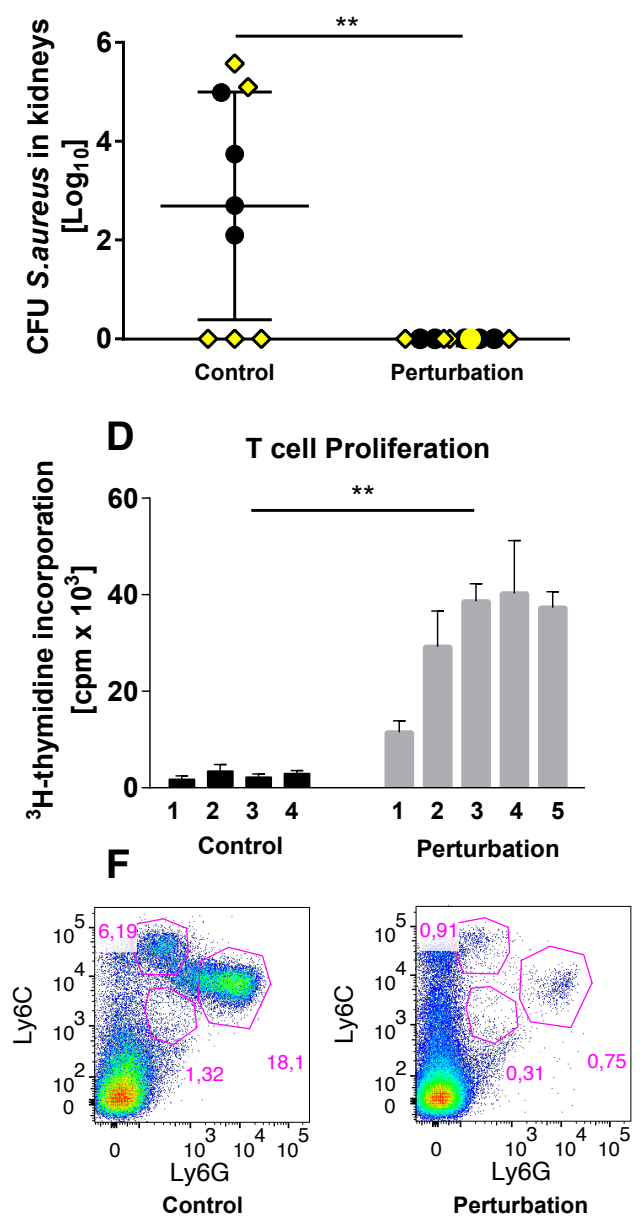

C HK treatment with S. pyogenes

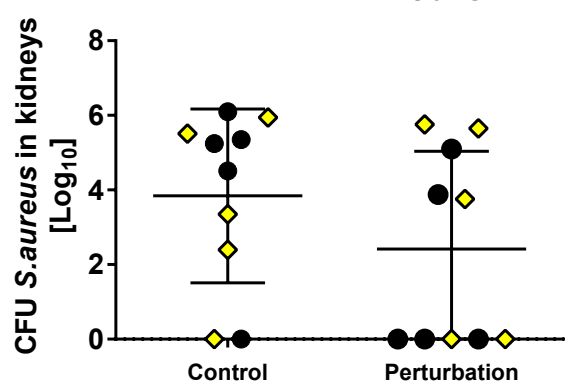

E T cell Proliferation

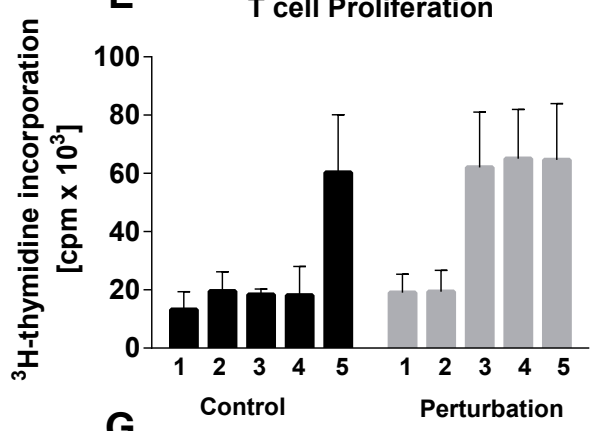

G
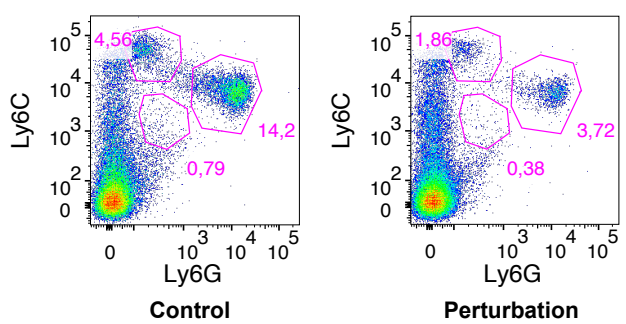

Figure 3: Experimental set up, bacterial loads in kidneys, $T$ cell proliferation and MDSC subsets. (A) Experiments started on day 0 with IV injection of $5 \times 10^{7} \mathrm{~S}$. aureus cells. On day $14,10^{8} \mathrm{HK}$ bacteria of $S$. aureus $(\mathbf{B}, \mathbf{D}, \mathbf{F})$ or $S$. pyogenes $(\mathbf{C}, \mathbf{E}, \mathbf{G})$ were injected intraperitoneally. On day 37 post-infection, sampling was conducted (Methods). Mice treated with HK bacteria injection accomplished sterilizing immunity (Methods). Success percentage was (B) 100\% for treatment with HK S. aureus bacteria and (C) $50 \%$ for treatment with HK S. pyogenes bacteria. In contrast, bacteria in the majority of control mice's kidneys persisted. (D-E) T cells in uncured mice remained suppressed, while T cells in cured mice after treated with (D) HK S. aureus bacteria or (E) HK S. pyogenes bacteria recovered their proliferative function (Methods). The numbers 1-5 represent each individual mouse from either the control or HK (Perturbation) group. (F-G) Percentage of each MDSC subset (monocytic-like CD11 b ${ }^{+} \mathrm{Ly}_{6 C^{+}} \mathrm{Ly}_{6} \mathrm{G}^{\text {low }}$ (M-MDSC), neutrophilic-like CD11b ${ }^{+} \mathrm{Ly}^{6 C^{\text {low }}} \mathrm{Ly} 6 \mathrm{G}^{+}$(PMN-MDSC), and eosinophilic-like CD11 b ${ }^{+}$Ly6C ${ }^{\text {low }}$ Ly6G ${ }^{\text {low }}$ (Eo-MDSC) MDSCs) in the spleens of mice that received PBS (Control) or HK (F) S. aureus and (G) S. pyogenes (Perturbation). All results were obtained from experiments in cohorts of five animals from two independent analyses represented by black bullets and yellow rhombuses. 
$\mathrm{CD} 11 \mathrm{~b}^{+}$Ly $6 \mathrm{C}^{+}$monocytes increased massively after the HK injection, whereas in case of HK S. pyogenes treatment, which cured half of the infected hosts, $\mathrm{CD} 11 \mathrm{~b}^{+} \mathrm{Ly} 6 \mathrm{G}^{+}$granulocytes (neutrophils) increased massively after the HK injection (Fig. S5).

\section{Reasoning for past unsuccessful applications of the treatment}

Our model-driven protocol suggesting the HK dose, its administration day, and day of complete clearance has been proven reliable and effective. Even though administration of killed cells as treatment for infections has been used in the past, this kind of therapy has not been well established. This is due to lacking information regarding the HK dose needed and day(s) of administration that could resolve the infection successfully. At the moment all treatments involving inactivated bacteria have been based on vague experimental experience.

Here, we explain in silico why heat- or formalin-killed bacteria treatments used so far have not been successful in yielding clearance. We base our arguments on a previous study ${ }^{32}$, where scientists administered at least 19 formalin-killed bacteria injections with increased dose over the period of 3 months in human patients with furunculosis.

In the study none of the chronically infected patients was reported to have attained sterilizing immunity, even though they experienced moderate to strong clinical improvement. The injecting scheme in the study consisted of increasing HK doses $\left(B_{d}\right)$ given in intervals of 3-5 days as following:

- Suspension I: $(0.1,0.2,0.3,0.4,0.5) \times 5 \times 10^{8}$

- Suspension II: $(0.3,0.4,0.5,0.6,0.7,0.8,0.9,1) \times 10^{9}$

- Suspension III: $(0.5,0.6,0.7,0.8,0.9,1) \times 2.5 \times 10^{9}$.

We assumed that the bacterial capacity in humans is 1000 times greater than the bacterial capacity in mice, created the corresponding murine suspensions:

- Suspension I: $(0.1,0.2,0.3,0.4,0.5) \times 5 \times 10^{5}$

- Suspension II: $(0.3,0.4,0.5,0.6,0.7,0.8,0.9,1) \times 10^{6}$

- Suspension III: $(0.5,0.6,0.7,0.8,0.9,1) \times 2.5 \times 10^{6}$,

and applied them in our mouse model in silico every 4 days, starting from day 14, when chronic infection is established in mice.

The conventional administration of injections is based on the belief that repeated vaccination could work more efficiently. Here, we employed in silico the analogous protocol that has been used in humans and showed that repeated administration of injections with increasing dose cannot render eradication of the infective agent (Fig. 4, (A,B,C)). We also explored the case where repeated injections of fixed dose are given. However, even with high fixed doses the treatments still fail to eliminate the infection (Fig. 4, (D,E,F)). Our results explicitly negate the current belief by showing that challenging the system repeatedly and in high frequency intervals does not result in cure (Fig. 4).

We also correlated the HK dose, number of injections and intervals [days] between injections for HK doses from $10^{6}$ to $10^{7}$ (Fig. S6). Our in silico results suggested that the suspensions and intervals used in the study with furunculosis patients ${ }^{32}$ were fruitless attempts towards bacterial clearance, since the administered HK doses in intervals of 3-5 days could not reactivate the hosts' immune systems in a sufficiently strong manner against bacteria (Fig. S6). We furthermore associated the HK dose, number of injections and intervals [days] between injections for HK doses from $10^{7}$ to $10^{8}$. Our results clearly indicated that the longer the intervals between injections, the higher the HK doses required for cure and the lesser the probability for cure (Fig. S7).

Finally, we investigated how the administration time of a HK injection affects the clinical outcome. Even though treatments with low HK dose cannot confer complete clearance of bacteria, they can still alleviate the infection. In fact, administration of the treatment as early as possible, leads to longer remission of the infection (Fig. S8A). Lastly, for middle-doses the day of administration is crucial for the outcome of the infection, since it can provide cure if given as early as possible, or not affect the infection state (Fig. S8B). 

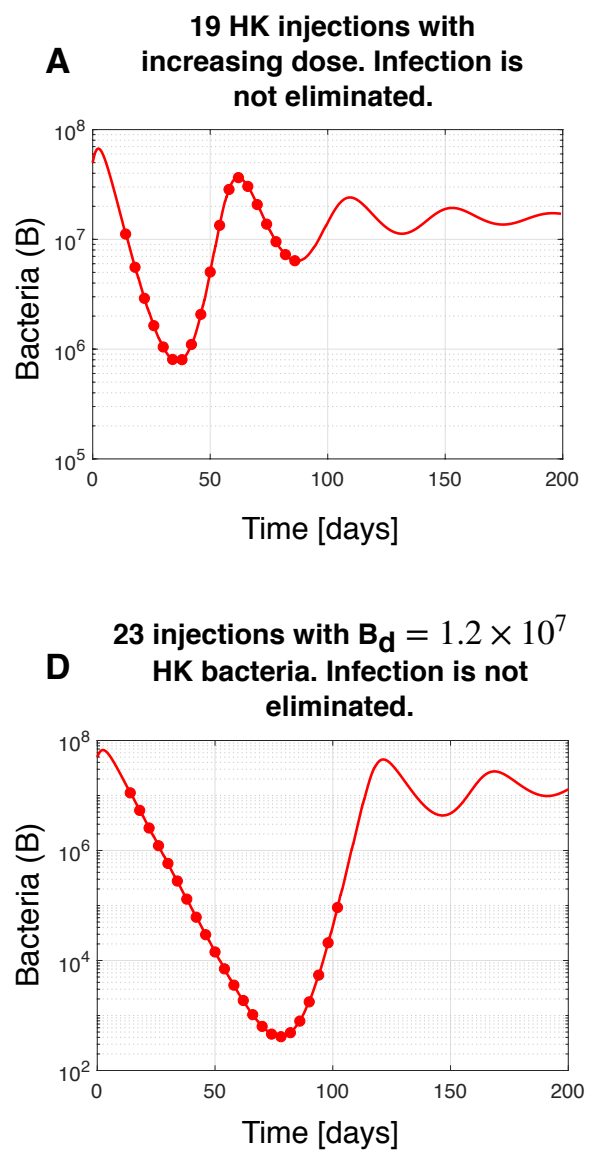
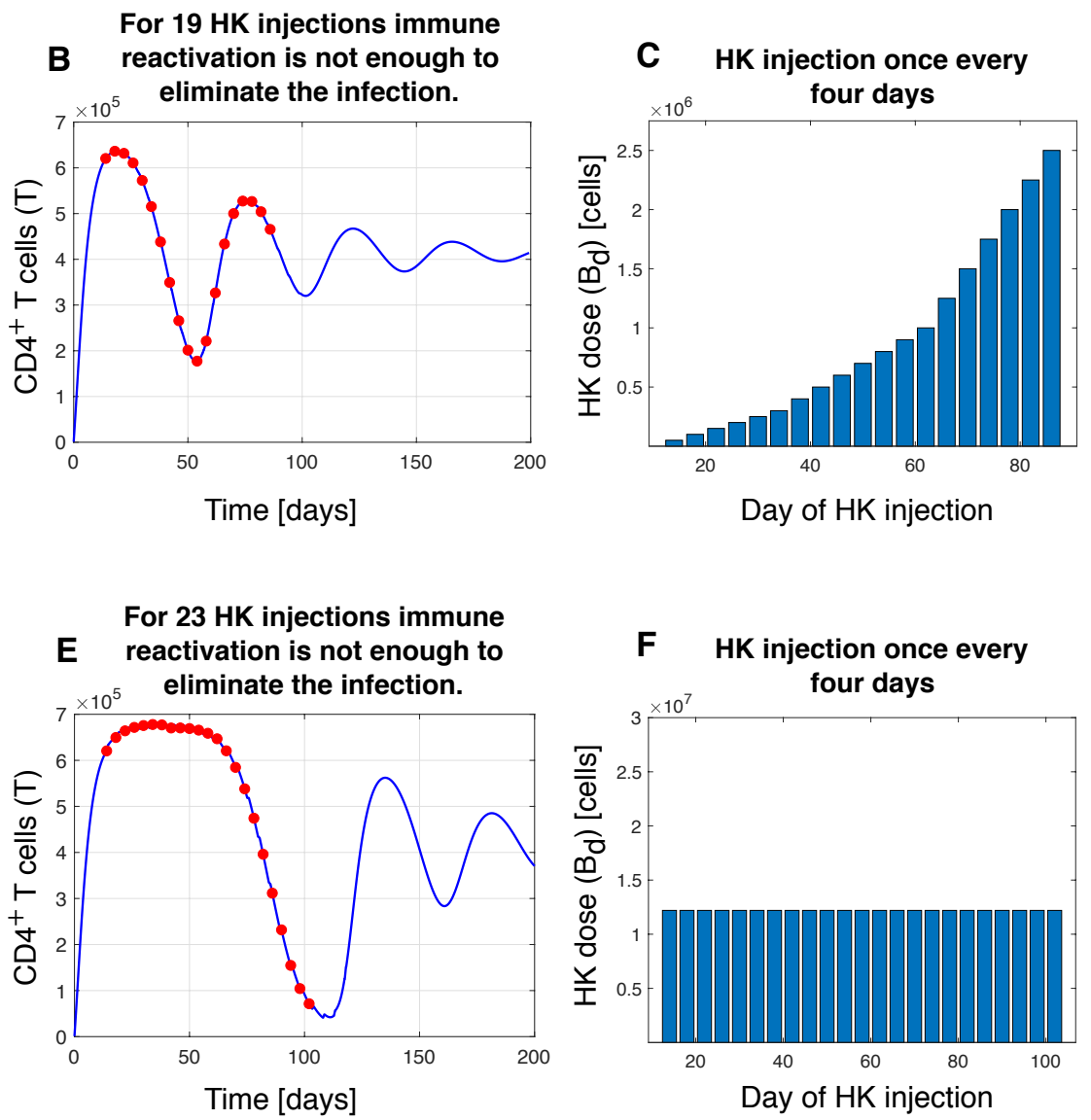

Figure 4: Repeated administration of dead bacteria injections cannot render clearance. (A, B, C) Nineteen HK bacteria injections are administered with increasing dose once every four days starting from day 14 post-infection in an analogous way as reported in humans. However, the infection still persists. (D, E, F) Twenty-three HK bacteria injections are administered with fixed dose once every four days starting from day 14 post-infection. The fixed dose is almost tenfold higher than the highest dose in the murine suspensions (i.e. $\left.2.5 \times 10^{6}\right)$. Still, the infection persists.

\section{Discussion}

To date no treatment has proven completely effective in resolving $S$. aureus chronic infections. There is no vaccine against $S$. aureus chronic infections because all clinical trials have failed ${ }^{33}$. The development of new antibiotic drugs could be a solution, however likely a temporary one, until the bacterium develops anew mechanisms of resistance. Knowing what a plague S. aureus chronic infections are, also that a slew of people suffer from such recalcitrant diseases, new ways of treatment are absolutely essential to find.

To escape the chronic phase of infection and result in sterilizing immunity, our model suggests four perturbation strategies, two of which are counter-intuitive and one validated in vivo. The analysis of the mathematical model suggests that by instantly heightening bacterial growth $\left(r_{b}\right)$, restricting bacterial killing by $\mathrm{T}$ cells $\left(c_{b}\right)$, boosting the immune system $\left(k_{b}\right)$, and/or reducing MDSC-suppression on T cells $(\Sigma)$, the stable steady state of established chronicity is perturbated and confers cure. The first two strategies would let bacteria grow in such level that the immune system would be reactivated, allowing bacterial eradication and clearance. The last two strategies are rather intuitive. In fact, targeting MDSCs ${ }^{34,35}$ and boosting the immune system ${ }^{32}$ have been shown to favour bacterial reduction but nonetheless failed to induce sterilizing immunity in chronically infected individuals. However, although MDSC-depletion seems to be beneficial in diseases such as cancer ${ }^{36 ; 37}$, in bacterial infections such treatment would simultaneously deplete important monocytes and by extension, dendritic cells and macrophages ${ }^{38}$.

Our model uses current knowledge in the field and refines it to foster complete clearance. All four suggested perturbation strategies, even so different from each other, have a common factor: during a chronic infection they initiate an acute inflammation, which instigates proinflammatory responses. Consequently, the immune system 
is impelled to action, which further leads to alleviation of the infection or, given the adequate strength of stimulation, to sterilizing immunity. Our experiments in vivo verified that $\mathrm{HK}$ injection initiates acute inflammation

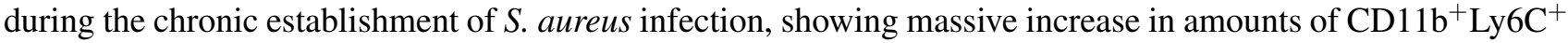
monocytes and $\mathrm{CD} 11 \mathrm{~b}^{+} \mathrm{Ly}_{6 \mathrm{G}}^{+}$granulocytes (neutrophils) in HK treated mice (Fig. S5).

Blood samples taken twelve hours after the HK injections with $S$. aureus or $S$. pyogenes showed significant reduction of leukocytes, confirming the effect of the treatments. However, S. aureus HK treatment also reduced significantly monocytes, neutrophils, and platelets, in contrast to HK $S$. pyogenes treatment, verifying that HK treatment with $S$. aureus is more effective than with $S$. pyogenes. Change in lymphocyte numbers was insignificant after both treatments (data not shown).

For the experiments regarding antigen-specificity, S. pyogenes was chosen because together with $S$. aureus they are the two most common gram-positive cocci of medical significance ${ }^{39}$. Interestingly, HK administration of the $S$. pyogenes antigen, still cured half of the infected hosts. This could possibly happen as a result of trained immunity, a de facto immune memory of the innate immune system, which is believed to empower a stronger immune response upon a subsequent inflammatory stimulus ${ }^{40}$. To clarify this possibility, future work could be to experiment with infected mice deprived of T cells (e.g. Rag, SCID, or nude mice) and observe their response to our HK treatment protocols. The 50\% sterilizing immunity in HK S. pyogenes treated mice (Fig. 3C) may also imply that a non-antigen specific HK perturbation should be combined with other perturbation strategies (antigen specific or not) to be completely effective. Nevertheless, our in silico results suggest that a higher HK $S$. pyogenes dose alone could have eliminated more MDSCs and hence could have reduced T cell suppression to a higher extent. The freed T cells would have then be able to exert their aggressive effect on bacteria and possibly also completely resolve the infection (Fig. S4). Future experiments could be conducted to test these hypotheses.

The hallmark of our model is that it can predict how each perturbation strategy may provide the sufficient intensity of stimulation that is necessary to yield complete clearance (Figs. 2C, S2-S3). Although the results of this study are limited to the specific mouse line of C57BL/6 female mice, the in silico results can be easily obtained when fitting the parameters of the mouse line or human of interest. This is because the model analysis was based on the model's non-dimensionalized form, which allows for the parameter values to change easily, before proceeding to the numerical analysis.

Applying treatment with HK bacteria has not only enhanced T cell activation, as we originally aimed and expected, but has also led to reduction of MDSCs and hence their inhibitory effect on T cells. Consequently, in the separatrix in Fig. 2A, the infected mouse condition did not improve by an upwardly vertical movement, as expected, but rather a diagonal left movement. This indicates that in bacterial infections autovaccination targets indirectly MDSCs, a fact that stayed until now unknown. It is hence likely that treated mice were cured some days before the scheduled sampling day. Future work could be to add a differential equation describing the population of MDSCs in time.

Additionally, we investigated why treatments using killed bacteria have not provided cure in the past. Heator formalin-killed bacteria treatments are narrowly established in medicine because there is no specific protocol stating the exact dose, number of injections and time between injections that would guarantee sterilizing immunity. Our analysis suggested that numerous injections with increasing dose or fixed dose of killed cells cannot render cure (Fig. 4) if the dose given does not exceed the threshold of effective dose that is required for clearance. Interestingly our study demonstrated that sterilizing immunity can be achieved with just one or only a few injections with high dose of HK bacteria (Fig. S7). Our interpretation is that HK treatments lead to cure when they succeed in initiating a strong immune response. Multiple injections that contain a low HK dose do not accomplish a sufficient immune reactivation that can resolve the infection, even if they are administered over a long period of time.

Our proposed HK injection protocol verified experimentally that sterilizing immunity can be achieved by only one injection. However, according to our analysis more injections with a lesser HK dose could still result in sterilizing immunity, if the intervals between the injections are short (Figs. S6-S7). Furthermore, the shorter the interval between treatments, the quicker the cure is achieved (data not shown).

The model also suggests that the sequence of the injection dates plays a major role in the outcome of the infection. For injections containing equal HK doses administered in different days post-infection, the infection is resolved in one case but remains unresolved in the other case (Fig. S8). This indicates that escaping from the chronic state does not only depend on the HK dose itself but also depends on the day of administration of the HK injection. This result adds to the usefulness of our model, since such knowledge would be laboriously deducted, if at all, by mere experience. 
It has been shown that $S$. aureus is becoming increasingly dangerous every year. It has been estimated that in 2001 S. aureus infections afflicted 292045 US hospital inpatients, caused almost 12000 inpatient deaths and cost $\$ 9.5$ billion in excess charges in US hospitals alone ${ }^{41}$. By 2014, the number of US inpatients afflicted with Methicillin-Susceptible and Methicillin-Resistant S. aureus alone has risen dramatically to 616070 individuals and associated costs were estimated to be around $\$ 14.6$ billion $^{42}$. Therefore finding new treatments against S. aureus infections is absolutely essential. Taken together, our study provides protocols for safe treatments and cure and can be translated as a strong basis for developing treatment protocols against $S$. aureus chronic infections in humans.

\section{Methods}

\section{Mathematical model}

The mathematical model applies to the chronic, non-acute infections caused by $S$. aureus. It comprises of currently known interactions between bacteria $\mathrm{B}(\mathrm{t})$, $\mathrm{T}$ cells $\mathrm{T}(\mathrm{t})$ and MDSCs $\Sigma$.

The ODE system reads

$$
\begin{aligned}
& \dot{B}(t)=r_{b} B(t)\left(1-\frac{B(t)}{\kappa}\right)-c_{b} T(t) B(t), \\
& \dot{T}(t)=r_{t} T^{2}(t)+k_{b} B(t)-c_{T} B(t) T(t)-\Sigma \cdot T(t) .
\end{aligned}
$$

Immunosuppression driven by MDSCs can be achieved in two ways. On the one hand, the bacterium can activate the MDSCs, which are resident in the site of infection. This is expressed with the term $c_{T} B(t) T(t)$ and implies the local immunosuppression by MDSCs. On the other hand, the activation and expansion of MDSCs can take place systemically, as a generic protective mechanism of the organism against long-lasting strong inflammation or as a mechanism of the bacteria for persistence. This is represented with the term $\Sigma \cdot T(t)$, since MDSC-mediated immunosuppression on $\mathrm{T}$ cells requires direct cell-cell contact or cell-cell proximity ${ }^{30 ; 43}$. $\mathrm{T}$ cell proliferation is represented by the term $r_{t} T^{2}(t)$. This is because activated T cells secrete Interleukin 2 (IL-2), which induces cell cycle progression of $\mathrm{T}$ cells. In return, it creates a positive feedback loop for $\mathrm{T}$ cell proliferation, and hence the term $r_{t} T^{2}(t)$. The parameter $r_{b}$ represents the proliferation rate of bacteria in the presence of the innate immunity phenomonologically capturing control of bacterial expansion by logistic growth. The term $c_{b} T(t) B(t)$ represents the T-helper mediated killing rate of bacteria, since the concentration of effector $\mathrm{CD} 4^{+} \mathrm{T}$ cells is pivotal to the killing efficacy of actual effector cells, such as macrophages. In murine models of $S$. aureus renal abscesses it has been shown that the infection progresses towards chronicity is due to the gradual loss of functionality of effector $\mathrm{CD}^{+}{ }^{+} \mathrm{T}$ cells ${ }^{31}$. In the following the term $\mathrm{T}$ cells will refer to the effector $\mathrm{CD} 4^{+} \mathrm{T}$ cells unless otherwise stated. The mathematical model was implemented and simulated in MATLAB, see www.mathworks.com.

\section{Fitting curves and standard deviation of parameters}

The unknown parameters in the model were estimated in three steps. First, the carrying capacity of bacteria $\kappa$ was estimated based on the data reported in Fig. $5 \mathrm{~A}^{31}$, where $\mathrm{S}$. aureus infected Rag-deficient mice showed nearly constant level of S. aureus in kidney from day 7 till day 56. The mean of these data points was taken as the carrying capacity. Secondly, the growth rate of bacteria was estimated by solving the logistic growth equation

$$
r=\frac{\ln \left(\frac{P\left(\kappa-P_{0}\right)}{P_{0}(\kappa-P)}\right)}{t}
$$

where $\kappa$ is the carrying capacity, $P_{0}$ is the initial inoculation number of bacteria and $\mathrm{P}$ is the bacterial CFU at time t. Our previous experimental data showed that the bacterial loads in Rag-deficient mice reached $75 \%$ of the carrying capacity on day 2 and fluctuated afterwards, after intravenous inoculation with $7 \times 10^{7} \mathrm{CFU}$ S. aureus. Assuming that bacterial load reached $75 \%$ of the carrying capacity by day 1 or day 2 , we determined the high and low boundary of $r_{b}$ to be 0.636 and 0.318 , respectively. The average of the low and high boundary was taken as the bacterial growth rate in the next step. Finally, the rest of the unknown parameters were estimated by fitting the data reported in Fig. $8 \mathrm{~B}^{31}$, where the absolute number of $\mathrm{CD} 4+\mathrm{T}$ cells in peripheral lymph 
nodes was monitored. The fitting process used a Markov Chain Monte Carlo version of Differential Evolution algorithm ${ }^{44}$. Parameter $c_{T}$ appeared much smaller than other parameters in the initial investigation. We tested, in a second study, the possibility of fitting the same data with $c_{T}=0$. The fit quality remained the same, therefore we concluded that $c_{T}$ is zero. This deduction accords with experimental reports which show Extramedullary Haematopoiesis (EH) during persistent infections ${ }^{45}$. Fitting curves are shown in Fig. S9 and fitted parameter values in Table 1.

\begin{tabular}{clcc}
\hline Parameter & Description & Value, [Confidence interval] & Unit \\
\hline$r_{b}$ & Bacterial growth rate (in the presence of innate immunity) & 0.477 & days $^{-1}$ \\
$\kappa$ & Carrying capacity of bacteria & $1.132 \times 10^{8}$ & cells \\
$c_{b}$ & T cell-mediated killing rate of bacteria (per cell) & $9.937 \times 10^{-7},\left[8.65 \times 10^{-7}, 1.25 \times 10^{-6}\right]$ & days $^{-1}$ \\
$r_{t}$ & T cell proliferation rate (per cell) & $2.0955 \times 10^{-7},\left[1.04 \times 10^{-7}, 2.94 \times 10^{-7}\right]$ & days $^{-1}$ \\
$k_{b}$ & T cell activation and recruitment rate & $0.001509,[0.0011,0.0017]$ & days $^{-1}$ \\
$c_{T}$ & Local T cell suppression rate (per cell) & 0 & days $^{-1}$ \\
$\Sigma$ & MDSC-mediated suppression rate & $0.14393,[0.072,0.18]$ & days $^{-1}$ \\
\hline
\end{tabular}

Table 1: Model parameter values as used for model analysis. In square brackets is the $95 \%$ confidence interval of the parameters as derived by a Markov Chain Monte Carlo version of Differential Evolution algorithm ${ }^{44}$.

\section{Simulating the perturbation treatment}

To simulate the perturbation strategy, we incorporated for a perturbation window (e.g. 12 hours) another term $k_{b} \cdot B_{d}$ or $B_{d}$ into the ODE describing T cells (Eq. (2)), where $B_{d}=10^{8}$ cells is the dose of heat-killed bacteria and $k_{b}=0.001509$ [days $^{-1}$ ] as estimated during the fitting process (Table 1). However, since the new term describes the addition of bacteria, despite them being inactivated, one would suggest that the term should be incorporated into the bacterial ODE. One would also argue that addition of heat-killed bacteria would initiate an acute inflammation, and hence result in reduction of MDSCs, which are associated with chronic infections. To eliminate all doubts about the model's predictions and robustness, we integrated the term in all three suggested locations in ODEs as shown below.

If heat-killed bacteria treatment is integrated in the T cell ODE

$$
\begin{aligned}
& \dot{B}(t)=r_{b} B(t)-\frac{r_{b}}{\kappa} B^{2}(t)-c_{b} T(t) B(t), \\
& \dot{T}(t)=r_{t} T^{2}(t)+k_{b} B(t)-c_{T} B(t) T(t)-\Sigma \cdot T(t)+k_{b} \cdot B_{d},
\end{aligned}
$$

then cure is expected by day 34.5 .

If heat-killed bacteria treatment is integrated in the bacterial ODE

$$
\begin{aligned}
& \dot{B}(t)=r_{b} B(t)-\frac{r_{b}}{\kappa} B^{2}(t)-c_{b} T(t) B(t)+B_{d}, \\
& \dot{T}(t)=r_{t} T^{2}(t)+k_{b} B(t)-c_{T} B(t) T(t)-\Sigma \cdot T(t),
\end{aligned}
$$

then cure is expected by day 29.6 .

If heat-killed bacteria treatment diminishes the MDSC effect

$$
\begin{aligned}
& \dot{B}(t)=r_{b} B(t)-\frac{r_{b}}{\kappa} B^{2}(t)-c_{b} T(t) B(t), \\
& \dot{T}(t)=r_{t} T^{2}(t)+k_{b} B(t)-c_{T} B(t) T(t)-\left(\Sigma-B_{d}\right) \cdot T(t),
\end{aligned}
$$

then cure is expected by day 14.15 .

All of them revealed eradication of bacterial cells by day 37 post-infection, the experimental measurent day. The perturbation strategy of the $k_{b}$ increase, as shown in this study, was simulated utilizing the equations (3). 


\section{Experimental protocols}

\section{Bacteria}

S. aureus strain $\mathrm{SH} 1000^{46}$ was grown to Mid-Log phase in brain heart infusion medium (BHI, Roth, Karlsruhe, Germany) at $37^{\circ} \mathrm{C}$ with shaking (120 rpm), collected by centrifugation, washed with sterile PBS, and diluted to the required concentration. The number of viable bacteria was determined after serial diluting and plating on BHI-agar.

\section{Mice and infection}

A previously described chronic renal abscess infection model ${ }^{31}$ has been used in this study. Pathogen-free, 10 weeks-old C57BL/6 female mice were purchased from Harlan-Winkelmann (Envigo, Netherlands). All animals were provided with food and water ad libitum, and housed in groups of up to 5 mice per cage in individually ventilated cages. Mice were infected with $5 \times 10^{7} \mathrm{CFU}$ of S. aureus in $100 \mu 1$ of PBS via a tail vein and monitored on a daily basis for weight loss and sign of pain or distress. At specified times of infection, mice were sacrificed by $\mathrm{CO}_{2}$ asphyxiation and the bacterial load was enumerated in kidney homogenates by plating 10 -fold serial dilutions on blood agar plates. Spleens were removed, transformed in a single cell suspension and further processed for FACS and proliferation assays.

Blood samples were collected with EDTA-treated tubes and the differential blood count was done with 50 $\mu 1$ of blood using a VetScan HM5 Hematology Analyzer (Abaxis).

In vaccination experiments, infected mice were injected intraperitoneally at day 14 of infection with $10^{8}$ heat-killed bacteria of S. aureus strain SH1000 or S. pyogenes strain A20 in $200 \mu 1$ of PBS that were prepared by heating a bacterial suspension at $60^{\circ} \mathrm{C}$ for $1 \mathrm{~h}$. At $12 \mathrm{~h}$ postchallenge, mice were sacrificed and peritoneal exudate cells (PEC) were isolated from infected mice by lavage of the peritoneal cavity with $2 \mathrm{ml}$ sterile PBS. The lavage fluid was centrifuged, supernatants stored at $-20^{\circ} \mathrm{C}$ for subsequent cytokine analysis, and PEC resuspended in complete RPMI, stained and analyzed by flow cytometry (see below).

Animal experiments were performed in strict accordance with the German regulations of the Society for Laboratory Animal Science (GV- SOLAS) and the European Health Law of the Federation of Laboratory Animal Science Associations (FELASA). All experiments were approved by the ethical board Niedersächsisches Landesamt für Verbraucherschutz und Lebensmittelsicherheit, Oldenburg, Germany (LAVES; permit N. 18/2798).

\section{Flow cytometry analysis}

Cells were incubated with purified rat anti-mouse CD16/CD32 (BD Biosciences) for 5 min to block $\mathrm{Fc}$ receptors and then stained with antibodies against CD11b (BioLegend), Ly6C (BioLegend), Ly6G (Miltenyi Biotec) for 20 min at $4^{\circ} \mathrm{C}$. Labeled cells were measured by flow cytometry using a BDTM LSR II flow cytometer (BD Biosciences) and analyzed by FlowJo software.

\section{Proliferation assay}

Spleen cells were seeded into 96-well flat-bottom plates at $5 \times 10^{5}$ cells/well in $100 \mu$ l of complete RPMI medium and stimulated with $2 \mu \mathrm{g} / \mathrm{ml}$ of anti-CD3/anti-CD28 antibodies (Sigma-Aldrich) at $37^{\circ} \mathrm{C}$ and $5 \% \mathrm{CO}_{2}$. After 3 days of incubation, the cells were pulsed with $1 \mu \mathrm{Ci}{ }^{3} \mathrm{H}$-thymidine (Amersham) and harvested $18 \mathrm{~h}$ later on Filtermats A (Wallac) using a cell harvester (Inotech). The amount of ${ }^{3} \mathrm{H}$-thymidine incorporation was measured in a gamma scintillation counter (Wallac 1450; MicroTrilux).

\section{Statistical analyses}

All data were analyzed with GraphPad Prism 7.0. Comparisons between several groups were made using a parametric ANOVA test with Tukey post-test multiple comparison test. Comparison between two groups was performed using a t-test. $\mathrm{P}$ values $<0.05$ were considered significant. 


\section{Authors' Contributions}

LAP, HH, SK, GZ, and MMH designed the study, developed the methodology and interpreted the results. GZ performed the fitting process. LAP performed the model implementation and simulations, and analyzed the results. LAP and EM designed and conducted the experiments. LAP, KKD and IS analyzed the experimental results. HH and MMH supervised the study. All authors wrote the paper and approved the final version of the manuscript.

\section{Acknowledgements}

LAP was supported by the German Federal Ministry of Education and Research within the initiative e:Mednetwork of systems-medicine, project MultiCellML (FKZ: 01ZX01707C). SK was supported by the German Federal Ministry of Education and Research within the Measures for the Establishment of Systems Medicine, project SYSIMIT (BMBF eMed project SYSIMIT, FKZ: 01ZX1308B and by the Helmholtz Association, Zukunftsthema "Immunology and Inflammation" (ZT-0027). GZ was supported by the Helmholtz Association within the initiative Immunology and Inflammation, project "Aging and Metabolic Programming" (AMPro). HH was supported by the Helmholtz Initiative on Personalized Medicine - iMed. The authors thank Sabine Lehne and Lothar Gröbe for excellent technical assistance, and Anna Ntalli, Heiko Enderling and Andreas Papaxenopoulos for useful comments on a draft of this article.

\section{References}

[1] Vichitra Sukumaran and Sanjaya Senanayake. Bacterial skin and soft tissue infections. Australian prescriber, 39(5):159, 2016.

[2] Sarah A Horst, Verena Hoerr, Andreas Beineke, Carolin Kreis, Lorena Tuchscherr, Julia Kalinka, Sabine Lehne, Ina Schleicher, Gabriele Köhler, Thomas Fuchs, et al. A novel mouse model of staphylococcus aureus chronic osteomyelitis that closely mimics the human infection: an integrated view of disease pathogenesis. The American journal of pathology, 181(4):1206-1214, 2012.

[3] Kuo-Ti Peng, Ching-Chuan Hsieh, Tsung-Yu Huang, Pei-Chun Chen, Hsin-Nung Shih, Mel S Lee, and Pey-Jium Chang. Staphylococcus aureus biofilm elicits the expansion, activation and polarization of myeloid-derived suppressor cells in vivo and in vitro. PloS one, 12(8):e0183271, 2017.

[4] K Hiramatsu, Y Katayama, M Matsuo, T Sasaki, Y Morimoto, A Sekiguchi, and T Baba. Multidrug-resistant staphylococcus aureus and future chemotherapy. Journal of Infection and Chemotherapy, 20(10):593-601, 2014.

[5] Vilasack Thammavongsa, Hwan Keun Kim, Dominique Missiakas, and Olaf Schneewind. Staphylococcal manipulation of host immune responses. Nature Reviews Microbiology, 13(9):529-543, 2015.

[6] James Collins, Angus Buckling, and Ruth C Massey. Identification of factors contributing to t-cell toxicity of staphylococcus aureus clinical isolates. Journal of clinical microbiology, 46(6):2112-2114, 2008.

[7] C. Tebartz, S.A. Horst, T. Sparwasser, J. Huehn, A. Beineke, G. Peters, and E. Medina. A major role for myeloid-derived suppressor cells and a minor role for regulatory $\mathrm{T}$ cells in immunosuppression during Staphylococcus aureus infection. J. Immunol., 194(3):1100-1111, 2015.

[8] Anca Dorhoi and Nelita Du Plessis. Monocytic myeloid-derived suppressor cells in chronic infections. Frontiers in immunology, 8:1895, 2018.

[9] Oliver Goldmann, Andreas Beineke, and Eva Medina. Identification of a novel subset of myeloid-derived suppressor cells during chronic staphylococcal infection that resembles immature eosinophils. The Journal of infectious diseases, 216(11):1444-1451, 2017. 
[10] Je-In Youn and Dmitry I Gabrilovich. The biology of myeloid-derived suppressor cells: the blessing and the curse of morphological and functional heterogeneity. European journal of immunology, 40(11):2969$2975,2010$.

[11] Eva Medina and Dominik Hartl. Myeloid-derived suppressor cells in infection: A general overview. Journal of innate immunity, pages 1-7, 2018.

[12] Michael Ost, Anurag Singh, Andreas Peschel, Roman Mehling, Nikolaus Rieber, and Dominik Hartl. Myeloid-derived suppressor cells in bacterial infections. Frontiers in cellular and infection microbiology, $6,2016$.

[13] Thomas Vollbrecht, Renate Stirner, Amanda Tufman, Julia Roider, Rudolf M Huber, Johannes R Bogner, Andreas Lechner, Carole Bourquin, and Rika Draenert. Chronic progressive hiv-1 infection is associated with elevated levels of myeloid-derived suppressor cells. Aids, 26(12):F31-F37, 2012.

[14] Gang Ning, Lanhui She, Lirong Lu, Ying Liu, Yingfu Zeng, Ying Yan, and Chaoshuang Lin. Analysis of monocytic and granulocytic myeloid-derived suppressor cells subsets in patients with hepatitis c virus infection and their clinical significance. BioMed research international, 2015, 2015.

[15] Laura Strauss, Sabina Sangaletti, Francesca Maria Consonni, Gabor Szebeni, Sara Morlacchi, Maria Grazia Totaro, Chiara Porta, Achille Anselmo, Silvia Tartari, Andrea Doni, et al. Rorc1 regulates tumor-promoting “emergency" granulo-monocytopoiesis. Cancer Cell, 28(2):253-269, 2015.

[16] Ehsan Malek, Marcos de Lima, John J Letterio, Byung-Gyu Kim, James H Finke, James J Driscoll, and Sergio A Giralt. Myeloid-derived suppressor cells: The green light for myeloma immune escape. Blood reviews, 30(5):341-348, 2016.

[17] Chunqing Guo, Fanlei Hu, Huanfa Yi, Zhitao Feng, Changzheng Li, Lianjie Shi, Yingni Li, Hongjiang Liu, Xiaofei Yu, Hongxia Wang, et al. Myeloid-derived suppressor cells have a proinflammatory role in the pathogenesis of autoimmune arthritis. Annals of the rheumatic diseases, 75(1):278-285, 2016.

[18] Jun Dai, Mohamed El Gazzar, Guang Y Li, Jonathan P Moorman, and Zhi Q Yao. Myeloid-derived suppressor cells: paradoxical roles in infection and immunity. Journal of innate immunity, 7(2):116-126, 2015.

[19] RN Zadoks, HG Allore, TJ Hagenaars, HW Barkema, and YH Schukken. A mathematical model of staphylococcus aureus control in dairy herds. Epidemiology and infection, 129(02):397-416, 2002.

[20] Tian Ding, Young-Hwan Shim, Na-Jung Choi, Sang-Do Ha, Myung-Sub Chung, In-Gyun Hwang, and Deog-Hwan Oh. Mathematical modeling on the growth of staphylococcus aureus in sandwich. Food Science and Biotechnology, 19(3):763-768, 2010.

[21] Heeyoung Lee, Kyungmi Kim, Soomin Lee, Minkyung Han, and Yohan Yoon. Growth kinetics of staphylococcus aureus on brie and camembert cheeses. Journal of Dairy Research, 81(02):252-256, 2014.

[22] Emily Kajita, Justin T Okano, Erin N Bodine, Scott P Layne, and Sally Blower. Modelling an outbreak of an emerging pathogen. Nature Reviews Microbiology, 5(9):700-709, 2007.

[23] C Hogea, T Van Effelterre, and CJ Acosta. A basic dynamic transmission model of staphylococcus aureus in the us population. Epidemiology \& Infection, 142(3):468-478, 2014.

[24] ES McBryde, AN Pettitt, and DLS McElwain. A stochastic mathematical model of methicillin resistant staphylococcus aureus transmission in an intensive care unit: predicting the impact of interventions. Journal of Theoretical Biology, 245(3):470-481, 2007.

[25] Xiaoxia Wang, Sarada Panchanathan, and Gerardo Chowell. A data-driven mathematical model of camrsa transmission among age groups: evaluating the effect of control interventions. PLoS Comput Biol, 9(11):e1003328, 2013. 
[26] Erica D'Agata, Glenn F Webb, Mary Ann Horn, Robert C Moellering, and Shigui Ruan. Modeling the invasion of community-acquired methicillin-resistant staphylococcus aureus into hospitals. Clinical Infectious Diseases, 48(3):274-284, 2009.

[27] Alfonso J Rodriguez-Morales, Cruz N Rodriguez, Ada Garcia, Ivette Jimenez, Bileida Pastran, and Pilar Meijomil. Surveillance analysis of decreasing susceptibility of staphylococcus aureus to vancomycin using a mathematical model. International journal of antimicrobial agents, 29(5):607-609, 2007.

[28] Hwan Keun Kim, Vilasack Thammavongsa, Olaf Schneewind, and Dominique Missiakas. Recurrent infections and immune evasion strategies of staphylococcus aureus. Current opinion in microbiology, 15(1):9299, 2012.

[29] B. Brett Finlay and Grant McFadden. Anti-Immunology: Evasion of the Host Immune System by Bacterial and Viral Pathogensn. Cell, 124(4):767-782, 2006.

[30] Christina Tebartz, Sarah Anita Horst, Tim Sparwasser, Jochen Huehn, Andreas Beineke, Georg Peters, and Eva Medina. A major role for myeloid-derived suppressor cells and a minor role for regulatory $t$ cells in immunosuppression during staphylococcus aureus infection. The Journal of Immunology, 194(3):1100$1111,2015$.

[31] Christina Ziegler, Oliver Goldmann, Elias Hobeika, Robert Geffers, Georg Peters, and Eva Medina. The dynamics of $\mathrm{t}$ cells during persistent staphylococcus aureus infection: from antigen-reactivity to in vivo anergy. EMBO molecular medicine, 3(11):652-666, 2011.

[32] Silva Holtfreter, Joanna Jursa-Kulesza, Helena Masiuk, NJ Verkaik, Corné de Vogel, Julia Kolata, Monika Nowosiad, Leif Steil, Willem van Wamel, Alex van Belkum, et al. Antibody responses in furunculosis patients vaccinated with autologous formalin-killed staphylococcus aureus. European Journal of Clinical Microbiology \& Infectious Diseases, 30(6):707, 2011.

[33] Dominique Missiakas and Olaf Schneewind. Staphylococcus aureus vaccines: Deviating from the carol. Journal of Experimental Medicine, 213(9):1645-1653, 2016.

[34] Cortney E Heim, Debbie Vidlak, and Tammy Kielian. Interleukin-10 production by myeloid-derived suppressor cells contributes to bacterial persistence during staphylococcus aureus orthopedic biofilm infection. Journal of leukocyte biology, 98(6):1003-1013, 2015.

[35] Cortney E Heim, Debbie Vidlak, Tyler D Scherr, Curtis W Hartman, Kevin L Garvin, and Tammy Kielian. Il-12 promotes myeloid-derived suppressor cell recruitment and bacterial persistence during staphylococcus aureus orthopedic implant infection. The Journal of Immunology, 194(8):3861-3872, 2015.

[36] Viktor Fleming, Xiaoying Hu, Rebekka Weber, Vasyl Nagibin, Christopher Groth, Peter Altevogt, Jochen Utikal, and Viktor Umansky. Targeting myeloid-derived suppressor cells to bypass tumor-induced immunosuppression. Frontiers in immunology, 9:398, 2018.

[37] Livingstone Fultang, Silvia Panetti, Margaret Ng, Paul Collins, Suzanne Graef, Nagy Rizkalla, Sarah Booth, Richard Lenton, Boris Noyvert, Claire Shannon-Lowe, et al. Mdsc targeting with gemtuzumab ozogamicin restores t cell immunity and immunotherapy against cancers. EBioMedicine, 2019.

[38] Cortney E Heim, Debbie Vidlak, Tyler D Scherr, Jessica A Kozel, Melissa Holzapfel, David E Muirhead, and Tammy Kielian. Myeloid-derived suppressor cells contribute to staphylococcus aureus orthopedic biofilm infection. The Journal of Immunology, 192(8):3778-3792, 2014.

[39] Tomas Fiedler, Thomas Köller, and Bernd Kreikemeyer. Streptococcus pyogenes biofilms—formation, biology, and clinical relevance. Frontiers in cellular and infection microbiology, 5:15, 2015.

[40] Willem JM Mulder, Jordi Ochando, Leo AB Joosten, Zahi A Fayad, and Mihai G Netea. Therapeutic targeting of trained immunity. Nature Reviews Drug Discovery, page 1, 2019. 
[41] Gary A Noskin, Robert J Rubin, Jerome J Schentag, Jan Kluytmans, Edwin C Hedblom, Maartje Smulders, Elizabeth Lapetina, and Eric Gemmen. The burden of staphylococcus aureus infections on hospitals in the united states: an analysis of the 2000 and 2001 nationwide inpatient sample database. Archives of internal medicine, 165(15):1756-1761, 2005.

[42] Eili Y Klein, Wendi Jiang, Nestor Mojica, Katie K Tseng, Ryan McNeill, Sara E Cosgrove, and Trish M Perl. National costs associated with methicillin-susceptible and methicillin-resistant staphylococcus aureus hospitalizations in the united states, 2010-2014. Clinical Infectious Diseases, 68(1):22-28, 2018.

[43] Dmitry I Gabrilovich and Srinivas Nagaraj. Myeloid-derived suppressor cells as regulators of the immune system. Nature Reviews Immunology, 9(3):162-174, 2009.

[44] Cajo JF Ter Braak. A markov chain monte carlo version of the genetic algorithm differential evolution: easy bayesian computing for real parameter spaces. Statistics and Computing, 16(3):239-249, 2006.

[45] Chang H Kim. Homeostatic and pathogenic extramedullary hematopoiesis. Journal of blood medicine, $1: 13,2010$.

[46] Marie Jonsson, Staffan Arvidson, Simon Foster, and Andrzej Tarkowski. Sigma factor b and rsbu are required for virulence in staphylococcus aureus-induced arthritis and sepsis. Infection and immunity, 72(10):6106-6111, 2004. 


\section{${ }_{541}$ Supplementary material}

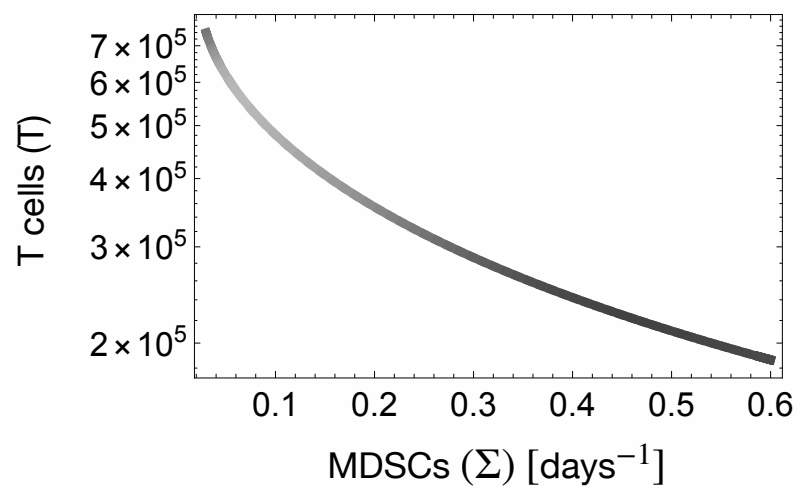

Figure S1: T cells and MDSCs exhibit an inverse proportional behaviour. Correlation between $\mathrm{T}$ cells and MDSC-mediated suppression is shown. The correlation was plotted using the analytical solutions of the $\mathrm{T}$ cell differential equation in steady state (Supplementary, Eq. S10) for increasing amounts of parameter $\Sigma$, representing the MDSCs. 
A

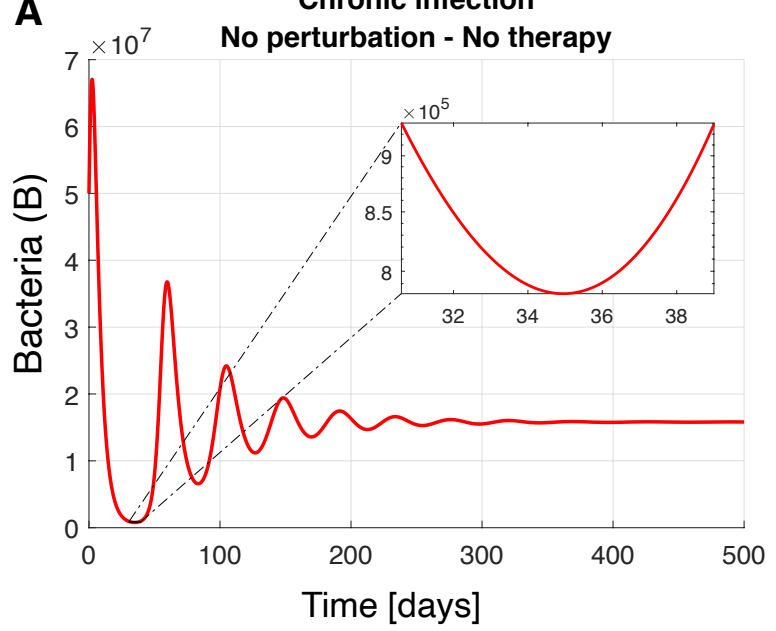

C $200 \% r_{b}$ increase on the 14th day of the infection

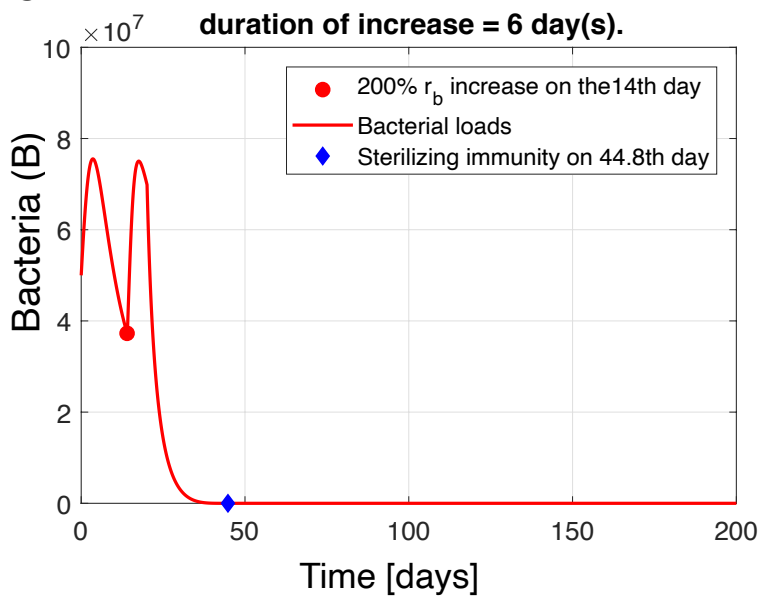

$30 \%$ MDSC reduction on the 14 th day of the infection

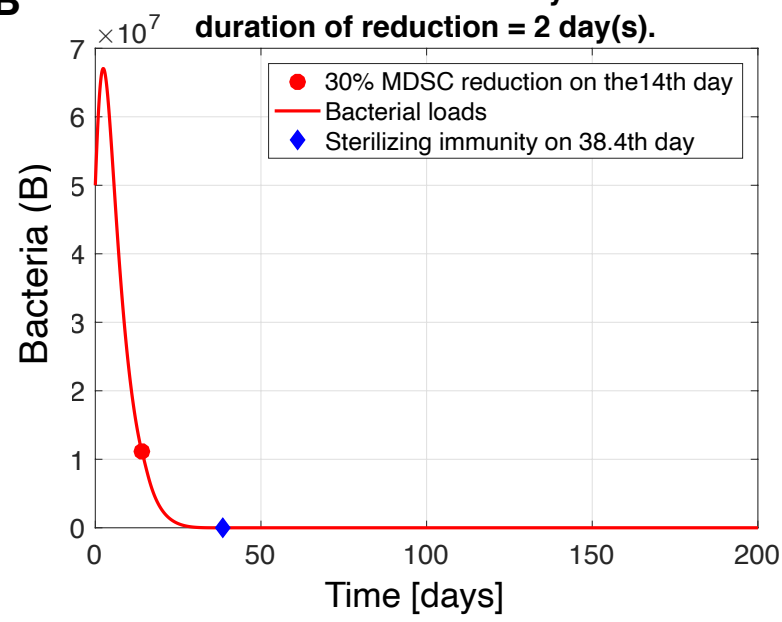

D $85 \% c_{b}$ reduction on the 14th day of the infection

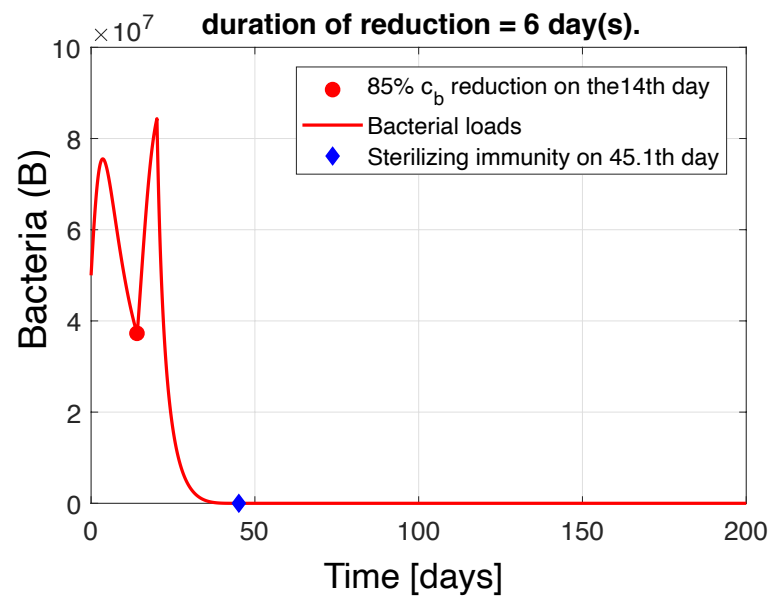

Figure S2: Perturbation treatments in silico suggest eradication of the bacterium. (A) Progression of $S$. aureus infection without perturbation treatment results in a stable state, clinically considered as chronic infection. The plotted bacterial dynamics in time is the numerical solution the ODE system (Eq. (1)). Chronic infection systems perturbed with treatments of either (B) diminished MDSC-mediated immunosuppression $(\Sigma)$ by $30 \%$, (C) increased bacterial growth $\left(r_{b}\right)$ by $200 \%$, or (D) decreased bacterial killing via T cells $\left(c_{b}\right)$ by $85 \%$, render sterilizing immunity in silico (represented with $\downarrow$ ). Treatments were applied on the 14th day post-infection (represented with $\bullet$ ) by decreasing or increasing the fitted value of the parameter of interest (Table 1) for a perturbation window of 2 or 6 days. 

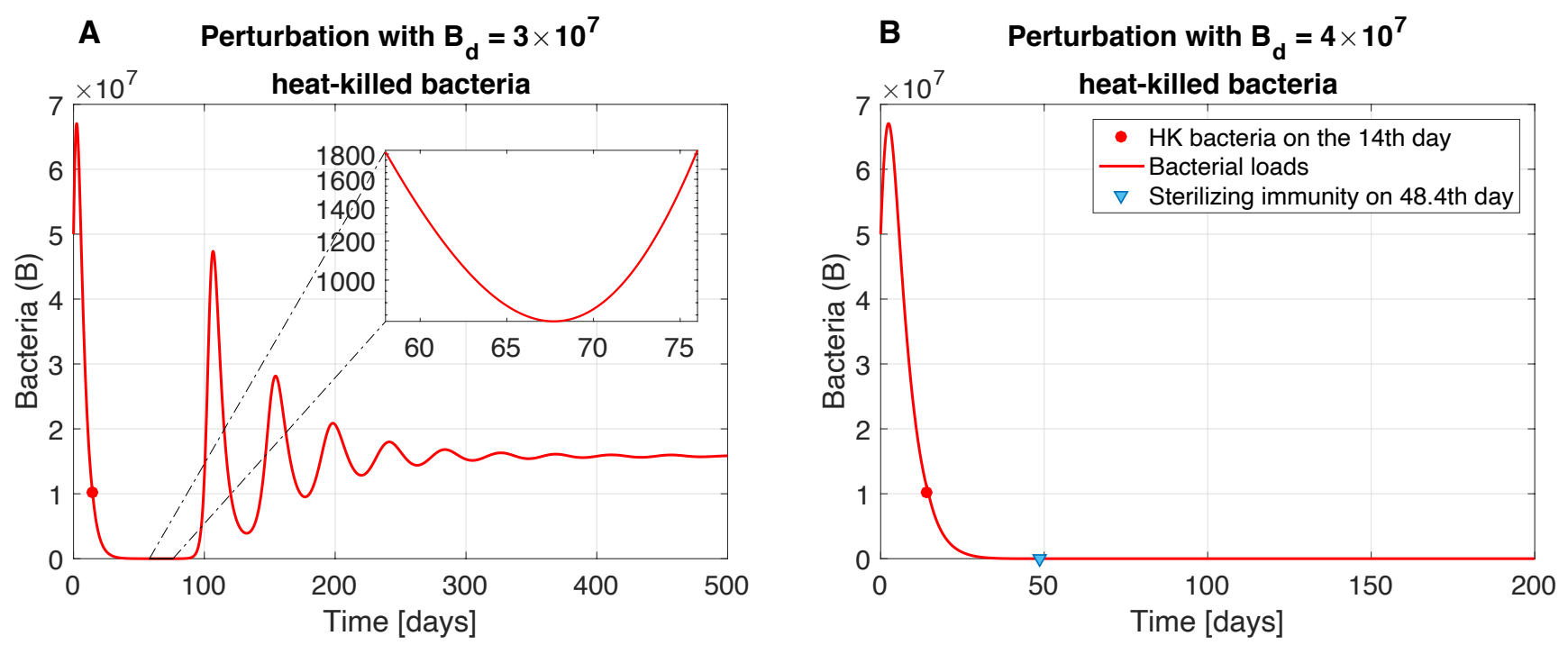

Figure S3: Minimum heat-killed dose $B_{d}$ required for sterilizing immunity. (A) Sterilizing immunity is not rendered when perturbating on day 14th (represented with $\bullet$ ) with dose of $B_{d}=3 \times 10^{7}$ heat-killed bacteria or less. However, (B) sterilizing immunity is attained by administering a heat-killed bacteria injection of minimum $B_{d}=4 \times 10^{7}$ cells. The treatments were administered in silico by adding the term $k_{b} \cdot B_{d}$ in the T-cell ODE at time $\mathrm{t}=$ Perturbation day for a 12-hour perturbation window (Methods).

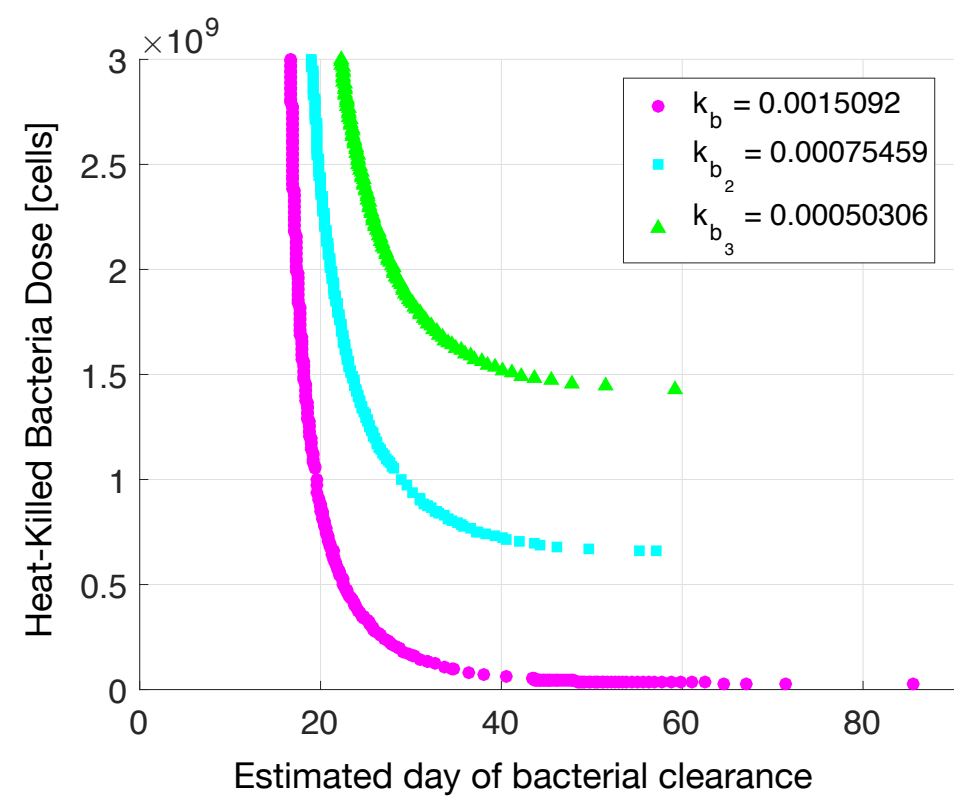

Figure S4: Heat-killed bacteria dose versus time of clearance. Stimulation of adaptive immune cells by heatkilled $S$. aureus cells happens with rate $k_{b}$ as defined during the fitting process (Table 1). Non-antigen specific stimulation means that streptococcal heat-killed bacteria stimulate the immune system with a lower rate than staphylococcal HK cells, e.g. $k_{b_{2}}=k_{b} / 2$ or $k_{b_{3}}=k_{b} / 3$. Simulation of treatment was done by adding the term $k_{b} \cdot B_{d}$ in the T cell ODE for a perturbation window of half day (Methods), where $k_{b}$ the immunostimulatory parameter and $B_{d}$ different doses of HK cells. The estimated day of clearance was defined the first time point when bacterial numbers $<0.000001$. 


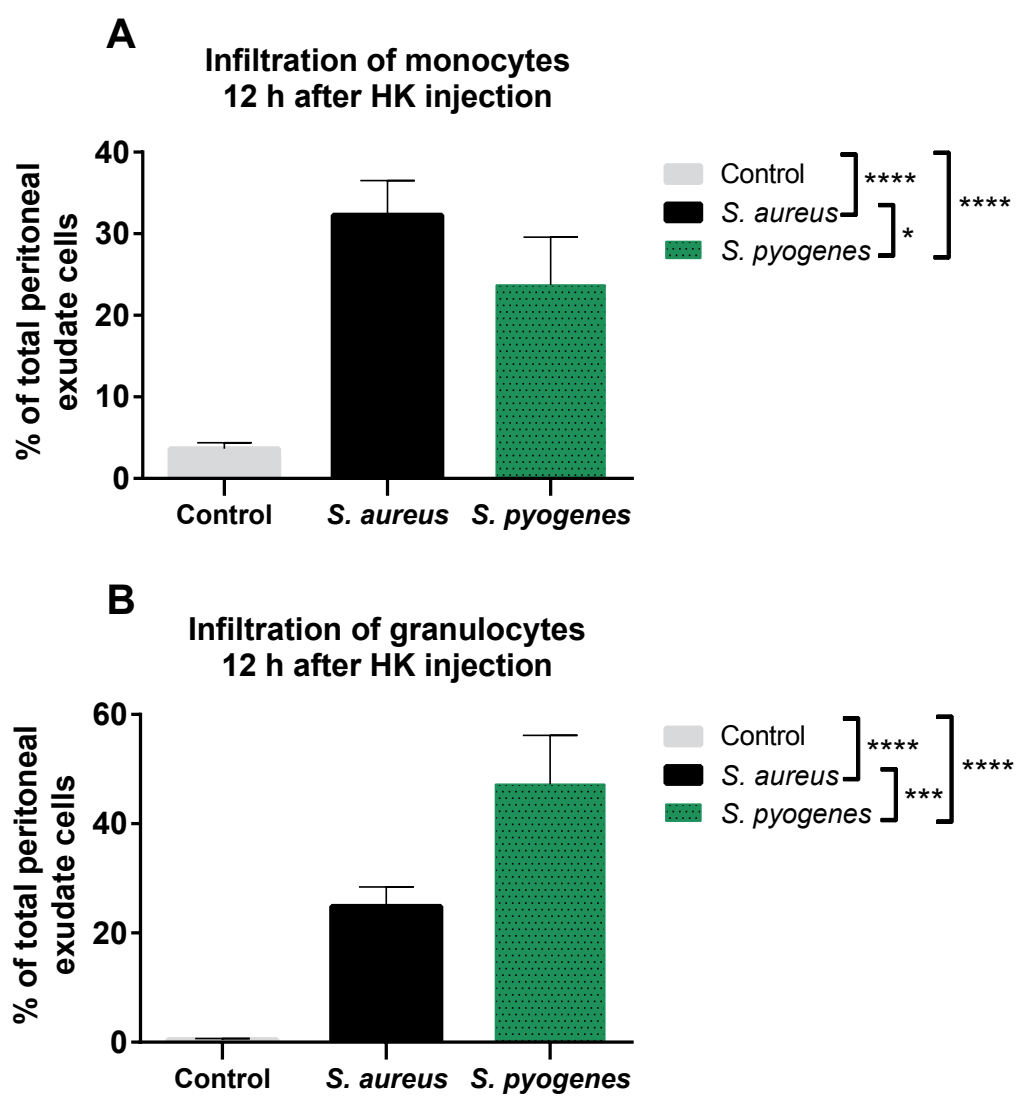

Figure S5: Infiltration of monocytes and granulocytes after intraperitoneal injection with HK $S$. aureus cells or $S$. pyogenes cells. Twelve hours after the administration of HK injection with $S$. aureus or $S$. pyogenes

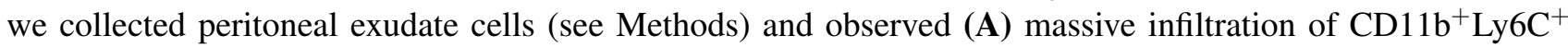
monocytes and (B) of CD11 b $\mathrm{Ly}^{+} \mathrm{G}^{+}$granulocytes (neutrophils). All results were obtained from cohorts of five animals from two independent experiments. 
A
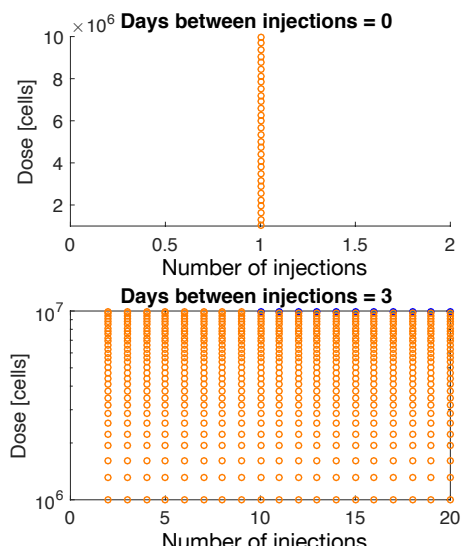

Number of injections

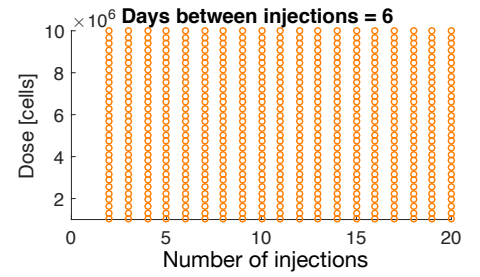

B

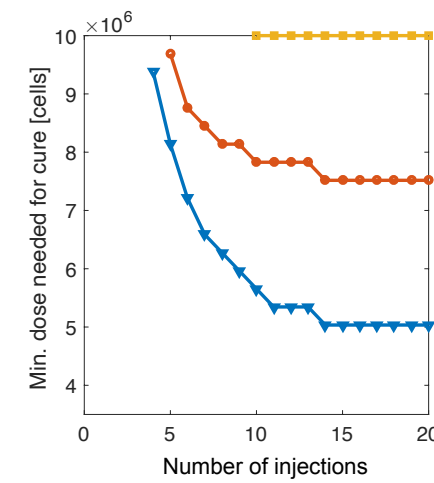

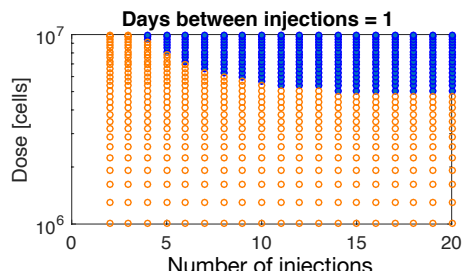

Number of injections

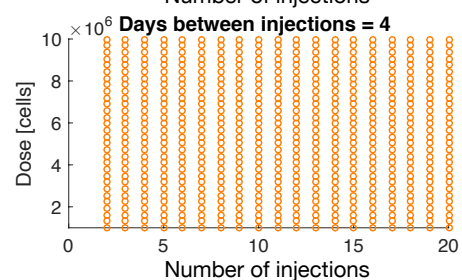

Number of injections

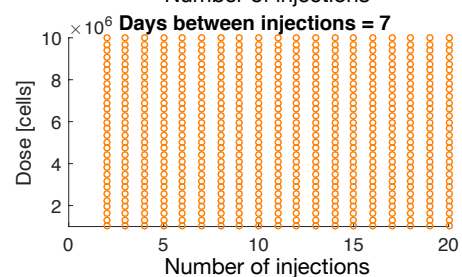

- Cure

- No cure
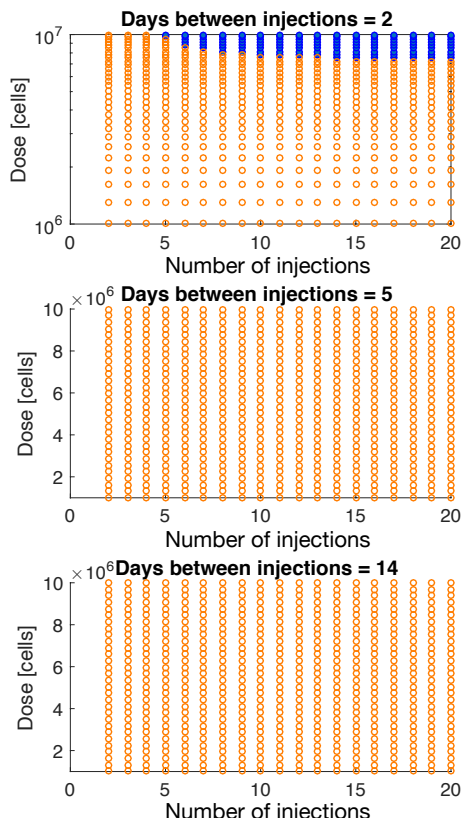

Intervals between injections [days]

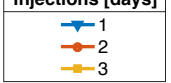

C

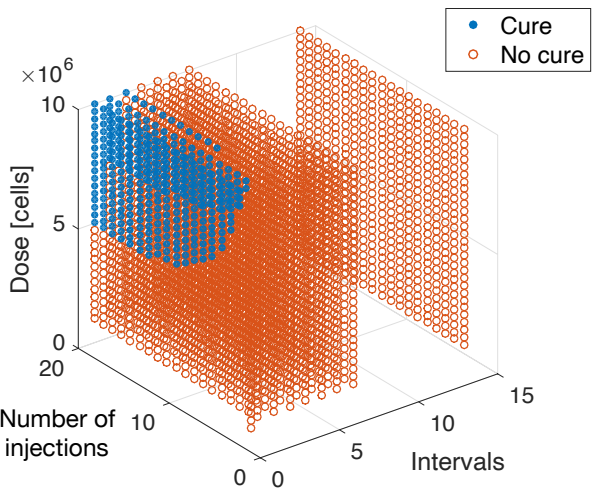

Figure S6: Correlation between HK doses, number of HK injections and intervals between injections. In silico predictions for cure plotted for HK doses ranging from $10^{6}$ to $10^{7}$, number of injections between 1 to 20 , and intervals between injections from 0 days to 7 days, or 2 weeks. For each injection day, the treatment was incorporated in the T cell ODE as $k_{b} \cdot B_{d}$, where $k_{b}$ the parameter defined in Table 1 and $B_{d}$ the administered dose (Methods). (A) Reduction of both the cure regime (blue) and possibility of cure with increasing intervals between HK injections. (B) Minimum HK dose needed for cure according to the number of HK injections and intervals between injections. For HK doses in the range $\left[10^{6}, 10^{7}\right]$ cure can be achieved only if the injections have intervals of 1, 2 or 3 days. (C) Three dimensional plot shows the "Cure" and "No cure" regions and their interconnection between HK dose, HK injections and intervals between injections. 

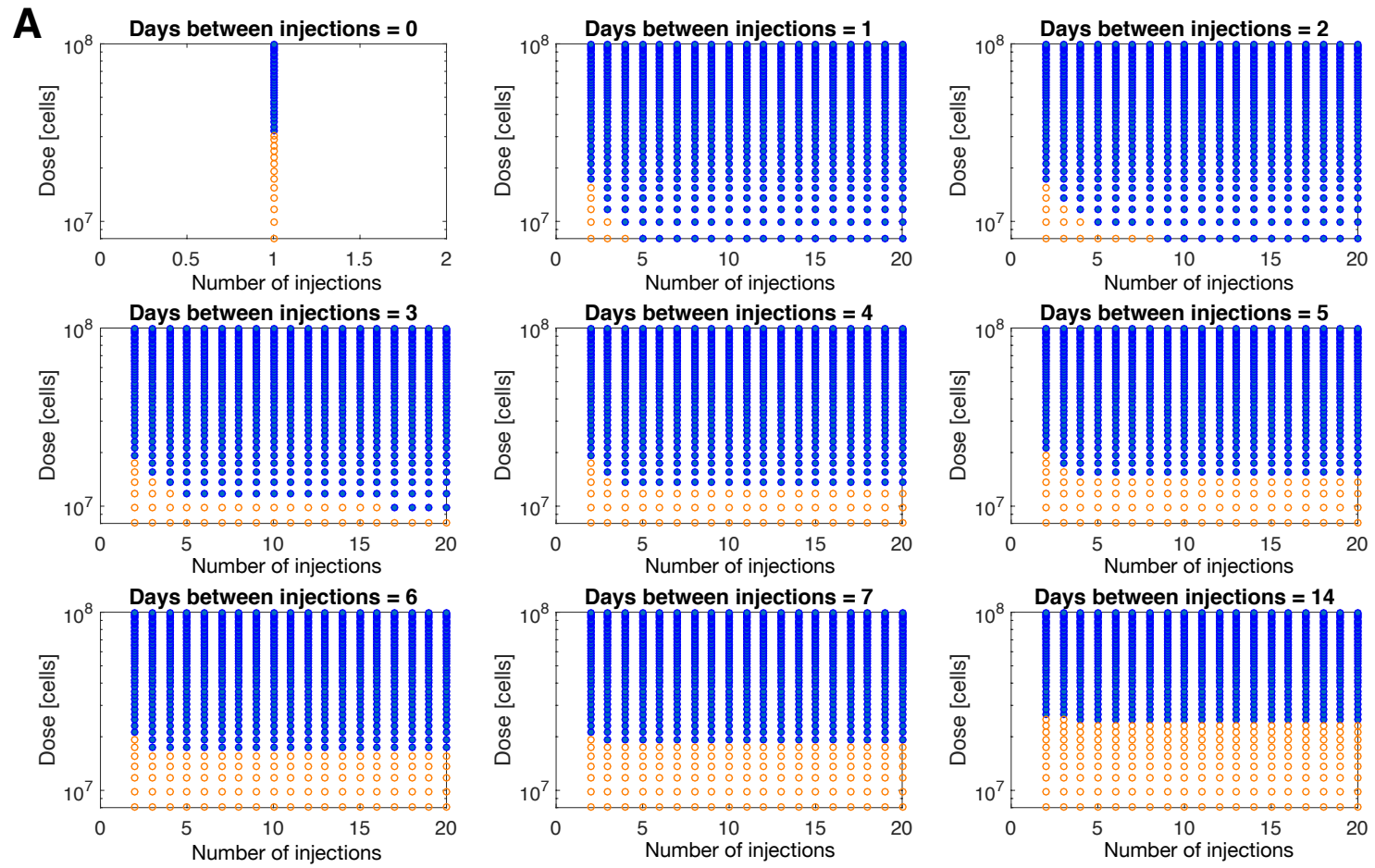

B

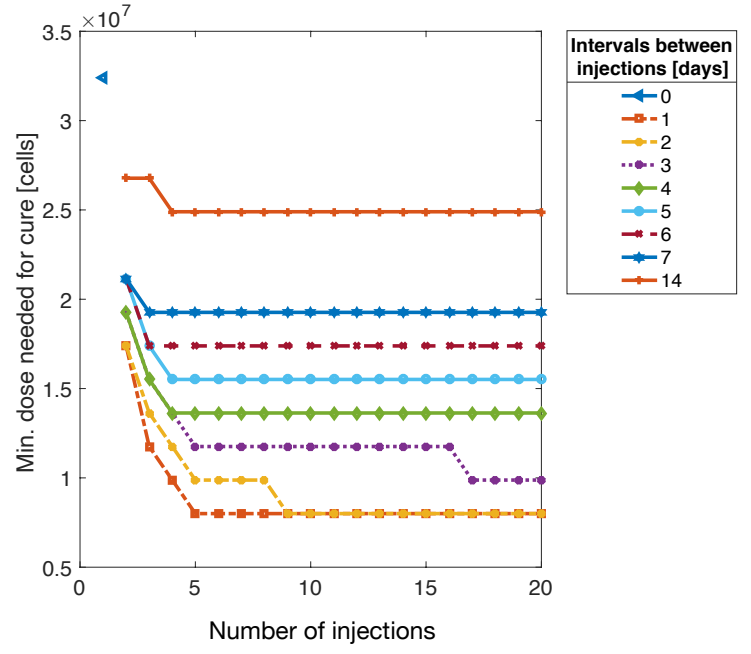

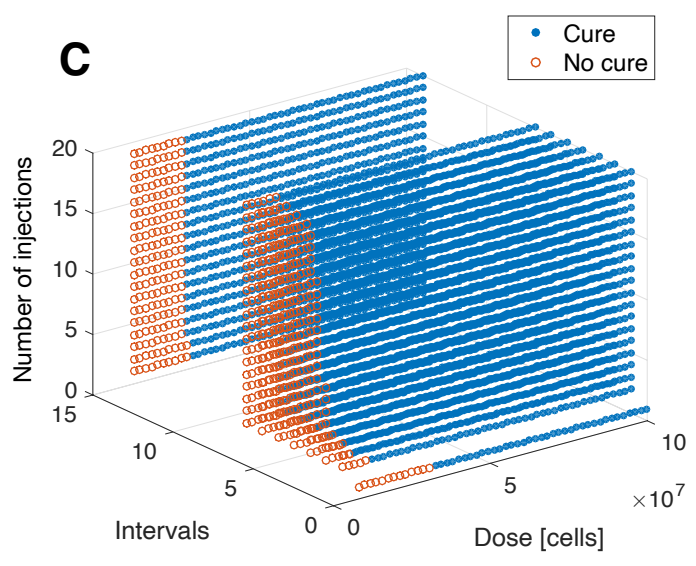

Figure S7: Same as in Fig. S6 for HK doses ranging from $10^{7}$ to $10^{8}$. 

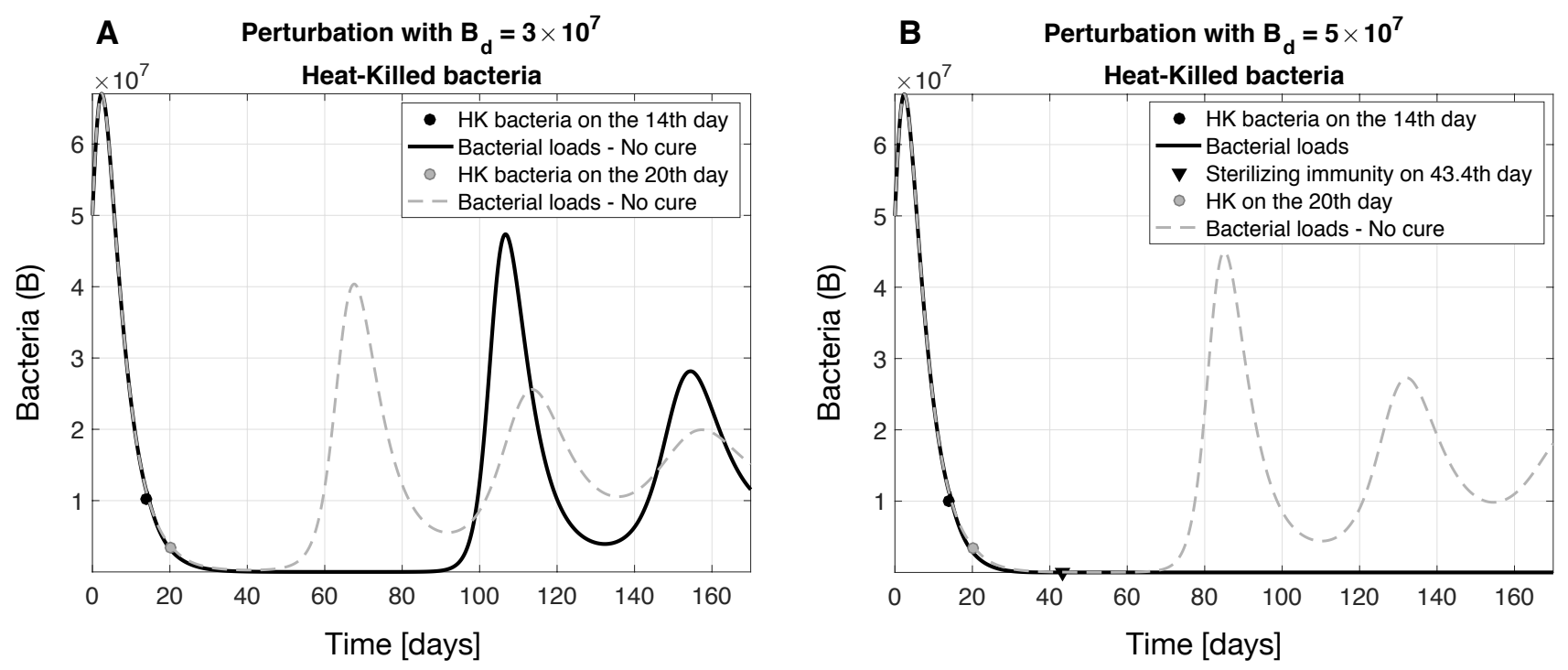

Figure S8: The administration time of the HK injection is crucial for the outcome of the infection. (A) Adminstration of HK injection with $B_{d}=3 \times 10^{7}$ cells is below the critical HK dose that is required for cure (estimated to be $4 \times 10^{7}$ in Fig. S3). However, HK injection on the 14th day post-infection confers longer remission of infection than when administrating the same HK dose on the 20th day post-infection. (B) Time of HK injection is decisive for the outcome of the infection. Adminstration of HK injection on the 14th day postinfection imparts sterilizing immunity, while administration of the HK injection on the 20th day post-infection does not affect the infection status. The HK treatment in silico was done by adding the term $k_{b} \cdot B_{d}$ in the T-cell ODE at time $\mathrm{t}=14$ or $\mathrm{t}=20$ for a 12-hour perturbation window (see Methods).
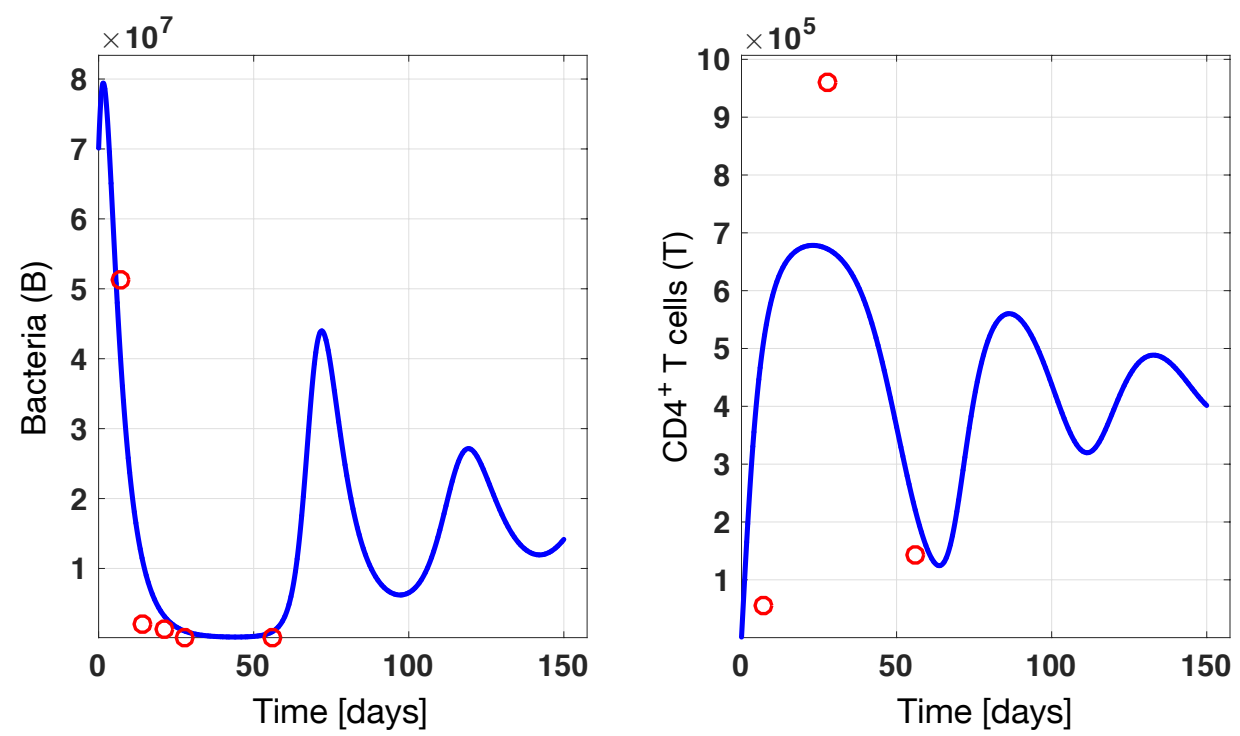

Figure S9: Plots for fitted model parameters $r_{t}, c_{T}, k_{b}, c_{b}$, and $\Sigma$ using a Markov Chain Monte Carlo version of Differential Evolution algorithm (Methods). Parameter values and confidence intervals are shown in Table 1.

\section{Scaling the model}

With the following change of variables we non-dimensionalize the ODE model (2).

$$
\begin{aligned}
B & =\beta_{0} \xi \\
T & =c_{0} \psi \\
t & =t_{0} \tau
\end{aligned} \Longrightarrow \frac{d B(t)}{d t}=\frac{\beta_{0} d \xi}{t_{0} d \tau}=r_{b} \beta_{0} \xi-\frac{r_{b}}{\kappa} \beta_{0}^{2} \xi^{2}-c_{b} c_{0} \psi \beta_{0} \xi
$$




$$
\Longrightarrow \frac{d \xi}{d \tau}=r_{b} t_{0} \xi-\frac{r_{b}}{\kappa} t_{0} \beta_{0} \xi^{2}-c_{b} t_{0} c_{0} \xi \psi
$$

Let's say that the coefficients of $\xi, \xi^{2}$ and $\xi \psi$ are equal 1 . Then

$$
t_{0}=\frac{1}{r_{b}}, \quad \beta_{0}=\kappa, \quad c_{0}=\frac{r_{b}}{c_{b}} .
$$

Therefore the new, non-dimensionalized equation for bacteria is

$$
\frac{d \xi}{d \tau}=\xi-\xi^{2}-\xi \psi
$$

For the equation $\dot{T}(t)$ we have the non-dimensionalized calculations:

$$
\begin{aligned}
& \frac{d T(t)}{d t}=\frac{c_{0} d \psi}{t_{0} d \tau}=r_{t} c_{0}^{2} \psi^{2}+k_{b} \beta_{0} \xi-c_{T} \beta_{0} \xi c_{0} \psi-\Sigma c_{0} \psi \\
\Longrightarrow & \frac{d \psi}{d \tau}=r_{t} t_{0} c_{0} \psi^{2}-\Sigma t_{0} \psi-c_{T} \beta_{0} t_{0} \xi \psi+\frac{k_{b} \beta_{0} t_{0}}{c_{0}} \xi
\end{aligned}
$$

Substitution of the $t_{0}, \beta_{0}$ and $c_{0}$ (found in Equation (S2)) gives the scaled equation for T:

$$
\frac{d \psi}{d \tau}=\alpha \psi^{2}+\beta \xi-\gamma \xi \psi-\delta \psi
$$

where

$$
\alpha=\frac{r_{t}}{c_{b}}, \quad \beta=\frac{\kappa k_{b} c_{b}}{r_{b}^{2}}, \quad \gamma=\frac{\kappa c_{T}}{r_{b}}, \quad \delta=\frac{\Sigma}{r_{b}} .
$$

\section{Calculation of Equilibrium points}

From equation (S3) we have:

$$
\xi-\xi^{2}-\xi \psi=0 \Longrightarrow \xi=0, \quad \text { and } \quad \xi=1-\psi
$$

- For $\xi=0$ in equation (S6) we have:

$$
\frac{d \psi}{d \tau}=0 \Longrightarrow \alpha \psi^{2}-\delta \psi=0 \Longrightarrow \psi_{1}=0, \quad \text { and } \quad \psi_{2}=\frac{\delta}{\alpha}
$$

- For $\xi=1-\psi$ in equation (S6) in steady state we conclude that:

$$
\psi_{3,4}=\frac{\beta+\gamma+\delta \pm \sqrt{[-(\beta+\gamma+\delta)]^{2}-4 \beta(\alpha+\gamma)}}{2(\alpha+\gamma)}
$$

Consequently the system has four equilibrium points in total:

- $\left(\xi_{1}, \psi_{1}\right)=(0,0)$

- $\left(\xi_{2}, \psi_{2}\right)=\left(0, \frac{\delta}{\alpha}\right)$

- $\left(\xi_{3,4}, \psi_{3,4}\right)=\left(1-\psi_{3,4}, \psi_{3,4}\right)$ where $\psi_{3,4}$ is shown in equation (S10). 


\section{Existence of $\psi_{3,4}$}

Equilibrium points $\psi_{3,4}$ exist only when $\Delta=(\beta+\gamma+\delta)^{2}-4 \beta(\alpha+\gamma) \geq 0$, i.e. only when $\psi_{3,4}$ have no imaginary part.

$$
\begin{aligned}
& \text { Equivalently } \\
& \qquad \begin{array}{l}
(\beta+\gamma+\delta)^{2}-4 \beta(\alpha+\gamma) \geq 0 \\
\beta^{2}+\gamma^{2}+\delta^{2}+2 \beta \gamma+2 \beta \delta+2 \gamma \delta-4 \alpha \beta-4 \beta \gamma \geq 0 \\
\beta^{2}+\gamma^{2}+\delta^{2}-2 \beta \gamma-2 \beta \delta+2 \gamma \delta-4 \beta(\alpha-\delta) \geq 0
\end{array}
\end{aligned}
$$

$$
(-\beta+\gamma+\delta)^{2}-4 \beta(\alpha-\delta) \geq 0
$$

According to the sign of the term $(\alpha-\delta)$ in condition (S11), we investigate when the equilibrium points $\psi_{3,4}$ exist.

- If $\alpha-\delta \leq 0 \Longrightarrow \delta \geq a$ then the equilibria $\psi_{3,4}$ exist.

- If $0<\delta<\alpha$ then we deformulate $\Delta$ as follows:

$$
\Delta=(-\beta+\gamma+\delta-2 \sqrt{\beta} \sqrt{\alpha-\delta}) \cdot(-\beta+\gamma+\delta+2 \sqrt{\beta} \sqrt{\alpha-\delta})
$$

a) If $-\beta+\gamma+\delta \geq 0$ then for existence of $\psi_{3,4}$ we require $-\beta+\gamma+\delta-2 \sqrt{\beta} \sqrt{\alpha-\delta} \geq 0$. Then:

$$
\begin{gathered}
-\beta+\gamma+\delta-2 \sqrt{\beta} \sqrt{\alpha-\delta} \geq 0 \Longrightarrow \beta-(\gamma+\delta)+2 \sqrt{\beta} \sqrt{\alpha-\delta} \leq 0 \\
\sqrt{\beta}^{2}+2 \sqrt{\beta} \sqrt{\alpha-\delta}-(\gamma+\delta) \leq 0 \Longrightarrow \sqrt{\beta} \leq \frac{-2 \sqrt{\alpha-\delta} \pm \sqrt{4(\alpha-\delta)-4[-(\gamma+\delta)]}}{2} \\
\sqrt{\beta} \leq-\sqrt{\alpha-\delta}+\sqrt{\alpha+\gamma}
\end{gathered}
$$

Note: The solution $\sqrt{\beta} \leq-\sqrt{\alpha-\delta}-\sqrt{\alpha+\gamma}$ is exempt because by definition $\sqrt{\beta} \geq 0$.

b) If $-\beta+\gamma+\delta \leq 0$ then for existence of $\psi_{3,4}$ we require $-\beta+\gamma+\delta+2 \sqrt{\beta} \sqrt{\alpha-\delta} \leq 0$. Then:

$$
\begin{aligned}
&-\beta+\gamma+\delta+2 \sqrt{\beta} \sqrt{\alpha-\delta} \leq 0 \Longrightarrow \beta-(\gamma+\delta)-2 \sqrt{\beta} \sqrt{\alpha-\delta} \geq 0 \\
& \sqrt{\beta}^{2}-2 \sqrt{\beta} \sqrt{\alpha-\delta}-(\gamma+\delta) \geq 0 \Longrightarrow \sqrt{\beta} \geq \frac{2 \sqrt{\alpha-\delta} \pm \sqrt{4(\alpha-\delta)-4[-(\gamma+\delta)]}}{2} \\
& \sqrt{\beta} \geq \sqrt{\alpha-\delta}+\sqrt{\alpha+\gamma}
\end{aligned}
$$

Note: The solution $\sqrt{\beta} \geq \sqrt{\alpha-\delta}-\sqrt{\alpha+\gamma}$ is trivially exempt.

In summary, equilibrium points $\psi_{3,4}$ exist for the range of $\delta$ and $\sqrt{k_{b}}$ shown in Fig. S10.

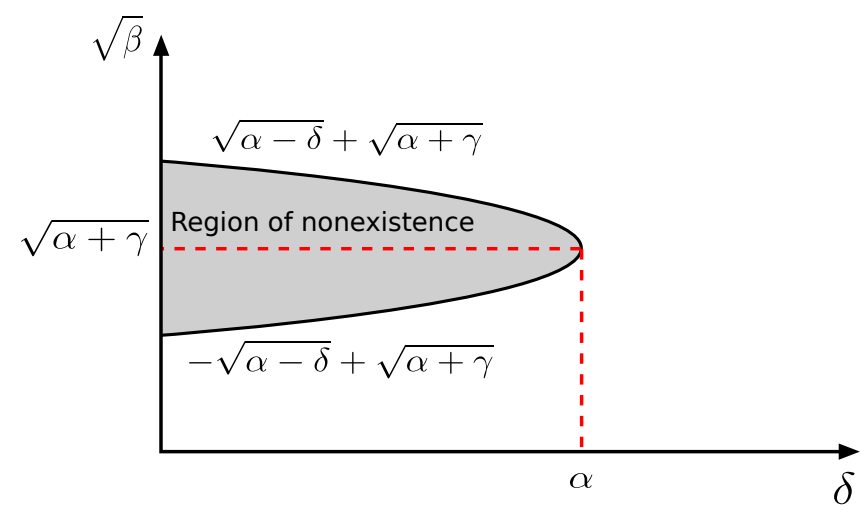

Figure S10: Existence of equilibrium points $\psi_{3,4}$. 


\section{Local stability analysis of equilibrium points}

Jacobian Matrix $J=\left[\begin{array}{ll}\frac{\partial \dot{\xi}}{\partial \xi} & \frac{\partial \dot{\xi}}{\partial \psi} \\ \frac{\partial \dot{\psi}}{\partial \xi} & \frac{\partial \dot{\psi}}{\partial \psi}\end{array}\right]=\left[\begin{array}{cc}1-2 \xi-\psi & -\xi \\ \beta-\gamma \psi & 2 \alpha \psi-\gamma \xi-\delta\end{array}\right]$

Evaluation of Jacobian matrix in the trivial equilibrium point $\left(\xi_{1}, \psi_{1}\right)$ :

$J(0,0)=\left[\begin{array}{cc}1 & 0 \\ \beta & -\delta\end{array}\right] \Longrightarrow|J(0,0)|=-\delta<0$

Since the determinant of the Jacobian matrix in equation (S16) is negative (i.e. det $=-\delta<0$ ), it means that the eigenvalues are of a different sign, and hence the trivial equilibrium point is unstable (i.e. saddle point, which is always unstable).

For the equilibrium point $\left(\xi_{2}, \psi_{2}\right)=\left(0, \frac{\delta}{\alpha}\right)$ we have:

$J\left(\xi_{2}, \psi_{2}\right)=\left[\begin{array}{cc}1-\frac{\delta}{\alpha} & 0 \\ \beta-\gamma \frac{\delta}{\alpha} & 2 \alpha \frac{\delta}{\alpha}-\delta\end{array}\right]=\left[\begin{array}{cc}1-\frac{\delta}{\alpha} & 0 \\ \beta-\frac{\gamma \delta}{\alpha} & \delta\end{array}\right] \Longrightarrow|J|=\delta \cdot\left(1-\frac{\delta}{\alpha}\right)$ for the equilibrium point $\left(\xi_{2}, \psi_{2}\right)$

The sign of the determinant depends on the term $\left(1-\frac{\delta}{\alpha}\right)$. When $\delta>\alpha$, the equilibrium point is saddle. This becomes unstable when $\delta<\alpha$. Next, we evaluate the Jacobian matrix in the equilibrium points $\left(\xi_{3,4}, \psi_{3,4}\right)$, by using $\xi=1-\psi \geq 0$,

$$
\begin{aligned}
|J(1-\psi, \psi)|=\left|\begin{array}{cc}
-1+\psi & -1+\psi \\
\beta-\gamma \psi & (2 \alpha+\gamma) \psi-(\gamma+\delta)
\end{array}\right| & =-(-1+\psi)(\beta+\delta+\gamma-2(\alpha+\gamma) \psi) \\
& =-(1-\psi)(2(\alpha+\gamma) \psi-(\beta+\delta+\gamma)) .
\end{aligned}
$$

We obtain the trace of the Jacobian matrix to determine the type of stability of equilibrium points $\left(\xi_{3,4}, \psi_{3,4}\right)$,

$$
\operatorname{tr}(J)=-1-\delta+\gamma(-1+\psi)+\psi+2 \alpha \psi=(1+2 \alpha+\gamma) \psi-(1+\gamma+\delta)
$$

We first need to find some critical values for $\psi$ :

- If $|J(1-\psi, \psi)|=0$, then

$$
\psi_{1}^{*}=\frac{\beta+\gamma+\delta}{2(\alpha+\gamma)}
$$

We know that the term $(1-\psi)$ equals $\xi$, which represents the bacteria, and hence $\xi=1-\psi \geq 0$.

- If $\operatorname{tr}(J)=0$, then

$$
\psi_{2}^{*}=\frac{1+\gamma+\delta}{1+2 \alpha+\gamma}
$$

From the critical points found in equations (S21) and (S22), the stability of the equilibrium points $\left(\xi_{3,4}, \psi_{3,4}\right)=$ $\left(1-\psi_{3,4}, \psi_{3,4}\right)$ can be classified as follows: 
- Stable node or spiral (Represents the chronic phase):

$$
\begin{aligned}
& |J| \geq 0 \\
& \operatorname{tr}(\mathrm{J}) \leq 0
\end{aligned} \Longrightarrow \begin{aligned}
& \psi_{1}^{*} \leq \frac{\beta+\gamma+\delta}{2(\alpha+\gamma)} \\
& \psi_{2}^{*} \leq \frac{1+\gamma+\delta}{1+2 \alpha+\gamma}
\end{aligned} \Longrightarrow \psi^{*} \leq \min \left\{\psi_{1}^{*}, \psi_{2}^{*}\right\}
$$

\section{- Saddle equilibrium point:}

$$
|J|<0 \Longrightarrow \psi_{1}^{*}>\frac{\beta+\gamma+\delta}{2(\alpha+\gamma)} \Longrightarrow \psi^{*}>\frac{\beta+\gamma+\delta}{2(\alpha+\gamma)}
$$

- Unstable node or spiral:

$$
\begin{aligned}
& |J| \geq 0 \\
& \operatorname{tr}(\mathrm{J}) \geq 0
\end{aligned} \Longrightarrow \begin{aligned}
& \psi_{1}^{*} \leq \frac{\beta+\gamma+\delta}{2(\alpha+\gamma)} \\
& \psi_{2}^{*} \geq \frac{1+\gamma+\delta}{1+2 \alpha+\gamma}
\end{aligned} \Longrightarrow \begin{aligned}
& \psi^{*} \leq \psi_{1}^{*} \\
& \psi^{*} \geq \psi_{2}^{*}
\end{aligned} \Longrightarrow \frac{\beta+\gamma+\delta}{2(\alpha+\gamma)} \geq \psi^{*} \geq \frac{1+\gamma+\delta}{1+2 \alpha+\gamma}
$$

However, since from fitting results (Table 1) $c_{T} \simeq 0$, we conclude that

$$
\psi_{1}^{*} \simeq \frac{\beta+\delta}{2 \alpha} \quad \text { and } \quad \psi_{2}^{*} \simeq \frac{1+\delta}{1+2 \alpha} .
$$

Assuming $\Sigma^{*}=\frac{r_{t} r_{b}}{c_{b}}$ and substituting $\alpha, \beta$, and $\delta$ from equations (S7),

$$
\psi_{2}^{*}=\frac{1+\frac{\Sigma}{r_{b}}}{1+\frac{2 r_{t}}{c_{b}}}=\frac{r_{b}+\Sigma}{r_{b}+2 \Sigma^{*}} .
$$

Since $\xi$ and $\psi$ are normalized, $\xi, \psi \geq 0$ and therefore $0 \leq \psi_{1}^{*}, \psi_{2}^{*} \leq 1$. Hence $\psi_{2}^{*} \leq 1$ resulting in

$$
\Sigma \leq 2 \Sigma^{*}
$$

Now

$$
\psi_{1}^{*}=\frac{\frac{\kappa k_{b} c_{b}}{r_{b}^{2}}+\frac{\Sigma}{r_{b}}}{2 \frac{r_{t}}{c_{b}}}=\frac{1}{2 \Sigma^{*}}\left(\frac{\kappa k_{b} r_{t}}{\Sigma^{*}}+\Sigma\right)
$$

From equation (S27)

$$
\psi_{1}^{*} \leq \frac{1}{2 \Sigma^{*}}\left(\frac{\kappa k_{b} r_{t}}{\Sigma^{*}}+2 \Sigma^{*}\right)=1+\frac{\kappa k_{b} r_{t}}{2\left(\Sigma^{*}\right)^{2}}>1
$$

As a consequence, $\psi_{1}^{*}$ is rejected and the stability analysis for the equilibrium points $\left(\xi_{3,4}, \psi_{3,4}\right)=\left(1-\psi_{3,4}, \psi_{3,4}\right)$ can be updated as follows:

\section{- Stable node or spiral (Represents the chronic phase):}

$$
\psi^{*} \leq \psi_{2}^{*} \Longrightarrow \psi^{*} \leq \frac{1+\gamma+\delta}{1+2 \alpha+\gamma}
$$

\section{- Unstable node or spiral:}

$$
\psi^{*} \geq \psi_{2}^{*} \Longrightarrow \psi^{*} \geq \frac{1+\gamma+\delta}{1+2 \alpha+\gamma}
$$

